\title{
THE ASYMPTOTIC EXPANSION OF INTEGRAL FUNCTIONS AND OF THE COEFFICIENTS IN THEIR TAYLOR SERIES
}

\author{
BY \\ E. M. WRIGHT
}

1. Introduction. Several authors have discussed the problem of deducing the asymptotic expansion of the integral function

$$
f(x)=\sum_{n=0}^{\infty} c_{0}(n) x^{n}
$$

for large $x$ from that of $c_{0}(n)$ for large $n$. References to most of the literature before 1936 are given in Ford's book [2](1) which contains a systematic account of the theory with improvements and simplifications. The most important early articles are due to Barnes [1] and Watson [7]. Barnes' methods have been developed and extended by Ford [2], Hughes [3] and Newsom $[5,6]$, while my own work $[11,14]$ is perhaps more closely connected with Watson's. In the present article I carry the theory a stage further by relaxing the conditions on $c_{0}(n)$ so that the class of functions $f(x)$ studied is considerably wider than before. We are led very naturally to this generalisation when we consider a new but closely associated problem, namely, that of deducing the asymptotic expansion for large $n$ of $c_{\lambda}(n)$, where $\lambda$ is any number and

$$
f(x+\lambda)=\sum_{n=0}^{\infty} c_{\lambda}(n) x^{n},
$$

from that of $c_{0}(n)$. Using the results which I obtain for the wider class of $f(x)$ I solve this second problem under suitable conditions on $c_{0}(n)$.

In what follows we use $R$ to denote the real part of a complex number and write

$$
\kappa^{\prime}=R(\kappa), \quad \rho=1 / \kappa, \quad \rho^{\prime}=R(\rho) .
$$

We always take $\kappa^{\prime}>0$, so that $\rho^{\prime}>0$ also. In [11] I supposed that, in the halfplane $R(\kappa t) \geqq K, c_{0}(t)$ is a regular function of $t$ and

$$
c_{0}(t)=\sum_{m=1}^{M} \frac{\kappa A_{m}}{\Gamma\left(\kappa t+\alpha_{m}\right)}+O\left(\frac{1}{\Gamma\left(\kappa t+\alpha_{M+1}\right)}\right)
$$

as $|t| \rightarrow \infty$. From these hypotheses I deduced the asymptotic expansion of $f(x)$ in all or part of the $x$-plane. Thus, if $\rho^{\prime}<1 / 2$, we have $f(x)=\sum G\left(x^{p}\right)$

Presented to the Society, December 31. 1947; received by the editors August 28, 1947.

(1) Numbers in brackets refer to the bibliography at the end of the paper. 
for all large $x$, where the summation extends over all values of $x^{\rho}$ for which $\left|\arg x^{\rho}\right|<\pi / 2$ and

$$
G(Y)=Y e^{Y}\left\{\sum_{m=1}^{M} A_{m} Y^{-\alpha_{m}}+O\left(Y^{-\alpha_{M+1}}\right)\right\} .
$$

If $\rho^{\prime} \geqq 1 / 2$, we cannot, without further information, find the asymptotic expansion of $f(x)$ throughout the whole $x$-plane. But, when there is a value of $x^{p}$ such that $\left|\arg x^{\rho}\right|<\pi / 2-\epsilon$ for some fixed $\epsilon>0$, we have $f(x)=G\left(x^{p}\right)$. Elsewhere in the $x$-plane the behavior of $f(x)$ for large $x$ does not depend solely on the behaviour of $c_{0}(t)$ for large $t$; the results are of a different kind from those with which we are concerned here and reference may be made to the full account in [11].

Results essentially equivalent to those of [11], but confined to real values of $\kappa$, were found independently by Hughes [3]. Our results include as particular cases most known expansions of special integral functions defined by Taylor series and have been used by Hughes [4] and by me $[9,10,12,13]$ to find the expansions of the generalized Bessel and hypergeometric functions. They do not give all the asymptotic properties of these functions, however; any "exponentially small" expansion has to be established by different methods (see, for example, $[10,12,13]$ ).

In a subsequent article [14], I supposed $c_{0}(t)$ to be regular and to satisfy (1.3) in a sector of the $t$-plane lying within the half-plane $R(\kappa t) \geqq K$ or, in the extreme case, to satisfy (1.3) only for all large, positive, integral(2) $t$. The same expansions of $f(x)$ are still valid, sometimes in the whole plane and sometimes in a more restricted region than before. The region of validity naturally depends on the sector of the $t$-plane in which $c_{0}(t)$ satisfies our hypotheses. By constructing suitable Gegenbiespiele, I showed that the restrictions on the region of validity are necessary.

The expansion of $c_{0}(t)$ postulated in (1.3) is less special than appears. We can deduce from the results of $[11,14]$ the asymptotic expansion of

$$
F(x)=\sum_{n=0}^{\infty} C_{0}(n) x^{n}
$$

where $C_{0}(t)$ is a regular function of $t$ and

$$
C_{0}(t)=t^{-\kappa} e^{g t}\left\{\sum_{m=1}^{M^{\prime}} B_{m} t^{\beta_{m}}+O\left(t^{\left.\beta_{M^{\prime}+1}\right)}\right\}\right.
$$

in an appropriate sector of the $t$-plane. If we write

$$
c_{0}(t)=\left(e^{\kappa-\sigma_{K}-\kappa}\right)^{t} C_{0}(t),
$$

(2) This corresponds to case (i) of Lemma 8 of [14]. It is not there stated that (1.3) need only be satisfied for integral $t$, but this is clear from the proof of the lemma. 
we have $F(x)=f\left(e^{\rho-\kappa} \kappa^{k} x\right)$ and

$$
c_{0}(t)=\left(\frac{e}{\kappa t}\right)^{\kappa t}\left\{\sum_{m=1}^{M^{\prime}} B_{m} t^{\beta_{m}}+O\left(t_{M^{\prime}}^{\beta^{\prime+1}}\right)\right\} .
$$

The well known asymptotic expansion

$$
\frac{1}{\Gamma(\kappa t+\alpha)}=\left(\frac{e}{\kappa t}\right)^{\kappa t} t^{1 / 2-\alpha}\left\{\sum_{l=0}^{L-1} A_{l}^{\prime} t^{-l}+O\left(t^{-L}\right)\right\}
$$

shows that (1.6) is equivalent to (1.3), provided the $M, \alpha_{m}$ and $A_{m}$ in (1.3) are suitably chosen. The interest of (1.3) lies in the simplicity of the relation between the coefficients in (1.3) and (1.4). This was discovered independently by Hughes [3] and by me [11].

In what follows the symbol $\sim$ is used in a more restricted sense than usual, so that $g_{1}(y) \sim g_{2}(y)$ denotes that $g_{1}(y)=g_{2}(y)\left\{1+O\left(y^{-K}\right)\right\}$ for some fixed $K>0$ as $|y| \rightarrow \infty$. There is never any ambiguity as to which variable (usually $x, t, Y$ or $n$ ) plays the part of $y$. We suppose that $f(x)$ is defined by (1.1), that

$$
c_{0}(t) \sim t^{\beta} e^{\psi(t)}(e / \kappa t)^{\kappa t}
$$

as $|t| \rightarrow \infty$ in a certain region in the $t$-plane and that, except when this region shrinks to a curve or to an enumerable infinity of points, $c_{0}(t)$ is regular in the region. In (1.8)

$$
\psi(t)=\sum_{j=1}^{J} a_{j} t^{b_{j}}
$$

where

$$
J \geqq 0, \quad 0 \leqq R\left(b_{j}\right)<1 .
$$

If $J=0$, the sum is empty and so $\psi(t)=0$. The restriction $0 \leqq R\left(b_{j}\right)$ is completely trivial since, if $R\left(b_{j}\right)<0$, exp $\left(a_{i} t^{t_{j}}\right) \sim 1$ for large $|t|$. Under these hypotheses I shall show that, in part of the $x$-plane,

$$
f(x) \sim 2^{1 / 2} \pi^{1 / 2} \kappa^{-1-\beta} X^{1 / 2+\beta} e^{P(X)},
$$

where $X$ is a particular value of $x^{p}$. Here $P(X)$ is the sum of a finite number of powers of $X$ and $P(X) \sim X$ as $|X| \rightarrow \infty$; in particular, if $R\left(b_{j}\right)<1 / 2$ for all $j, P(X)=X+\psi(\rho X)$. When $\rho^{\prime}<1 / 2$, I am able, under suitable conditions, to find a result valid for all large $x$.

It we replace (1.8) by a relation of the form

$$
c_{0}(t)=e^{\psi(t)}(e / \kappa t)^{\kappa t}\left\{\sum_{m=1}^{M} B_{m} t^{\beta_{m}}+O\left(t^{\beta_{M+1}}\right)\right\},
$$


we can replace (1.11) by

$$
f(x)=e^{P(X)}\left\{\sum_{m=1}^{M^{\prime}} B_{m}^{\prime} X^{\beta_{m}^{\prime}}+O\left(X^{\beta_{M+1}+1 / 2}\right)\right\}=I_{1}(X)
$$

(say). Unfortunately there is no simple relation between the coefficients in (1.12) and (1.13) of the kind existing between those in (1.3) and (1.4). For this reason and and to simplify my calculations, I confine myself to conditions of type (1.8) and results of type (1.11). But I state the more elaborate results in Theorem 4 and show (without proof) in $\$ 9$ how to calculate the $B_{m}^{\prime}$ and $\beta_{m}^{\prime}$ of (1.13) from the $B_{m}$ and $\beta_{m}$ of (1.12).

In the second part of the paper I use my results to study the $c_{\lambda}(n)$ of (1.2). If $c_{0}(t)$ satisfies $(1.8)$ in a suitable region, I show that

$$
c_{\lambda}(n) \sim c_{0}(n) e^{\lambda \psi(n, \lambda)} .
$$

Here $\psi(n, \lambda)$ is a polynomial in $\lambda$ of degree $\left(^{3}\right)\left[1 / \kappa^{\prime}\right]-1$, the coefficient of each power of $\lambda$ being a finite sum of (non-integral) powers of $n$ of order less than $n$; if $\kappa^{\prime}>1, \psi(n, \lambda)$ is always zero. If $c_{0}(t)$ satisfies (1.12) in the same region, we may replace (1.14) by a correspondingly more detailed result.

A particular case of some interest is that in which $c_{0}(t)$ has an expansion of the type of (1.6), so that $\psi(t)$ is an empty sum and $f(x)$ is a function of the class considered in $[11,14]$. If $\kappa^{\prime}>1$, so that $\psi(n, \lambda)=0$ for all $\lambda, c_{\lambda}(n)$ also has an expansion of the type of (1.6) with $t=n$, and $f(x+\lambda)$ belongs to the same class as $f(x)$. On the other hand, if $\kappa^{\prime}<1$, then $\psi(n, \lambda) \neq 0$ for $\lambda \neq 0$ and so $f(x+\lambda)$ belongs to the wider class of functions with which we are concerned in this paper. Hence, as soon as we study $c_{\lambda}(n)$, even for functions $f(x)$ of the restricted class previously discussed, we are led naturally to consider this wider class of $f(x)$.

There is a further interesting difference between the cases $\kappa^{\prime}>1$ and $\kappa^{\prime}<1$. When $\kappa^{\prime}>1$ (and when $\kappa=1$ ) the approximation (1.14) to $c_{\lambda}(n)$ may be almost trivially deduced, provided (1.8) is satisfied for $t=n, n+1, \cdots$. When $\kappa^{\prime}<1$, the position is entirely different; I am able to show by a Gegenbeispiel that (1.14) may be false even though (1.8) is true for all positive integral values of $t \geqq n$. Hence, when $\kappa^{\prime}<1$, I suppose that (1.8) holds for all large $t$ in a sector of positive (but arbitrarily small) angle having the positive half of the real axis in its interior; from this I deduce (1.14) but not trivially.

2. Notation. The numbers

$$
\kappa, \lambda, J, a_{1}, \cdots, a_{J}, b_{1}, \cdots, b_{J}
$$

are any numbers subject only to the restrictions that $J$ is a non-negative integer, that $\kappa^{\prime}>0$ and that (1.10) is satisfied. The sum $\psi(t)$ is defined by (1.9). As a minimum hypothesis, $c_{0}(t)$ satisfies (1.8) for all sufficiently large

(3) $[v]$ denotes the greatest integer not greater than $v$. 
positive integral values of $t$. These conditions are enough to ensure that the series in (1.1) converges for all $x$, so that $f(x)$ is an integral function. The values of the coefficients $c_{0}(n)$ in (1.1) are, of course, insufficient to determine the function $c_{0}(t)$ uniquely; this is unnecessary and the existence of any $c_{0}(t)$ satisfying our hypotheses and taking the value $c_{0}(n)$ of (1.1) at $t=n$ is sufficient for our purpose.

We use $\sum$ and $\Pi$ to denote summation and multiplication respectively over all integral $j$ such that $1 \leqq j \leqq J$. We write

$$
A_{j}=\rho^{b_{j}} a_{j}, \quad b=\max _{j} R\left(b_{j}\right), \quad c=\frac{1}{1-b}, \quad B=\max \left(b, 1-\kappa^{\prime}\right) .
$$

The number $s$ is always a positive integer and $r_{1}, \cdots, r_{J}$ are any set of nonnegative integers satisfying $\sum r_{j}=s$. We use $\sum_{(s)}$ to denote summation over all such sets $r_{1}, \cdots, r_{J}$ (where order is relevant) for the particular value of $s$ shown; if $J=0, \sum$ and $\sum_{(s)}$ denote empty sums whose value is zero. We write

$$
\tau=s-\sum r_{j} b_{j}, \quad T=\prod a_{j}^{r_{j} b_{j}^{r_{j}}\left(r_{j} !\right)^{-1},}
$$

where, as usual, $0 !=1$. It follows from the multinominal theorem that

$$
\frac{1}{s !}\left\{\psi^{\prime}(t)\right\}^{s}=\frac{1}{s !}\left(\sum a_{j} b_{j} t^{b_{j}-1}\right)^{s}=\sum_{(s)} T t^{-r} .
$$

We observe also that

$$
R(\tau) \geqq s(1-b)
$$

We write

$$
P(Y)=Y+\psi(\rho Y)+\sum_{s=2}^{[c]} \sum_{(s)}(1-\tau)^{s-2} T \rho^{8-\tau} Y^{1-\tau},
$$

so that, if $b<1 / 2$,

$$
P(Y)=Y+\psi(\rho Y)
$$

since $[c]=1$.

The number $\epsilon$, to be thought of as small, is any assigned positive number. $K$ is a positive number, not always the same at each occurrence, independent of the positive integers $n$ and $N$, the real variables $r, \theta, v$ and the complex variables $x, Y, u, t$, but possibly depending on some or all of the numbers $\epsilon, \mu_{1}, \mu_{2}$ and the numbers listed in (2.1). $K_{1}, K_{2}, \cdots$ are fixed numbers of the type $K$. The statement $g_{1}=O\left(g_{2}\right)$ denotes that there is a number $K$ such that $\left|g_{1}\right|<K\left|g_{2}\right|$ for all values of the variable in the region stated. All our statements are subject to the implied condition "for sufficiently large $|x|$ " (or $|t|,|Y|, n)$, except, of course, when we sum over $n$ as in (1.1). 
We write $\gamma=\arg \kappa$, where $-\pi / 2<\gamma<\pi / 2$, so that

$$
\kappa=\kappa^{\prime}(1+i \tan \gamma), \quad \rho=\rho^{\prime}(1-i \tan \gamma) .
$$

The numbers $\mu_{1}, \mu_{2}$ are any real numbers which satisfy $\left({ }^{4}\right)$

$$
-\pi / 2<-\mu_{1}<\gamma<\mu_{2}<\pi / 2 .
$$

We call the region in the $t$-plane in which

$$
|t|>K, \quad-\mu_{1} \leqq \arg (\kappa t) \leqq \mu_{2}
$$

the $\left(-\mu_{1}, \mu_{2}\right)$ sector. For convenience we apply the same name to the transform of this region in the $u$-plane, where $u=\kappa t$, namely the region in which

$$
|u|>K, \quad-\mu_{1} \leqq \arg u \leqq \mu_{2} .
$$

If $\rho^{\prime}<1 / 2$, we define $\mu_{0}$ and $\bar{\mu}_{0}$ by

$$
\begin{aligned}
\tan \mu_{0}=\frac{e^{2 \pi \rho^{\prime} \tan \gamma}-\cos 2 \pi \rho^{\prime}}{\sin 2 \pi \rho^{\prime}}, \quad \tan \bar{\mu}_{0}= & \frac{e^{-2 \pi \rho^{\prime} \tan \gamma}-\cos 2 \pi \rho}{\sin 2 \pi \rho^{\prime}} \\
& \left(\left|\mu_{0}\right|<\pi / 2,\left|\bar{\mu}_{0}\right|<\pi / 2\right) .
\end{aligned}
$$

By Lemma 3 of [14],

$$
\mu_{0}+\bar{\mu}_{0}=2 \pi \rho^{\prime},
$$

whence and from the definitions of $\mu_{0}, \bar{\mu}_{0}$ we have

$$
\cos \bar{\mu}_{0}=e^{2 \pi \rho^{\prime} \tan \gamma} \cos \mu_{0} .
$$

Hence

$$
\tan \pi \rho^{\prime} \tan \left(\mu_{0}-\bar{\mu}_{0}\right) / 2=\frac{\cos \bar{\mu}_{0}-\cos \mu_{0}}{\cos \bar{\mu}_{0}+\cos \mu_{0}}=\tanh \left(\pi \rho^{\prime} \tan \gamma\right)
$$

and

$$
\tan \left(\mu_{0}-\bar{\mu}_{0}\right) / 2=\tanh \left(\pi \rho^{\prime} \tan \gamma\right) \cot \pi \rho^{\prime} .
$$

Let $\gamma<\mu<\pi / 2$ and let $\omega=\omega(\mu, \gamma)$ be that root of the equation

$$
\cos \omega=\cos \mu \exp \left\{\left(\mu+\omega-2 \pi \rho^{\prime}\right) \tan \mu+2 \pi \rho^{\prime} \tan \gamma\right\}
$$

which satisfies $-\mu<\omega<\pi / 2$; by Lemma 1 (i) of [14] there is just one such $\omega$. We write

$$
\omega_{1}=\omega\left(\mu_{1},-\gamma\right), \quad \omega_{2}=\omega\left(\mu_{2}, \gamma\right) .
$$

The properties of $\omega, \omega_{1}$ and $\omega_{2}$ which we require are contained in the following lemmas. They are little more than restatements in our present notation of Lemmas 1 and 2 of [14].

(') In [14] we had $-\mu_{1} \leqq \gamma \leqq \mu_{2}$, but here we exclude equality. 
LEMMA 1. When $\gamma<\mu<\pi / 2, \omega(\mu, \gamma)$ is a one-valued continuous function of $\mu, \omega>-\mu$ and $\omega \rightarrow-\gamma$ as $\mu \rightarrow \gamma$.

LEMMA 2. (i) If $\rho^{\prime} \geqq 1 / 2$, $\omega$ increases steadily with $\mu$ and $\omega \rightarrow \pi / 2$ as $\mu \rightarrow \pi / 2$.

(ii) If $\rho^{\prime}<1 / 2$, $\omega$ increases steadily with $\mu$ when $\gamma<\mu<\mu_{0}$, attains its maximum value $\bar{\mu}_{0}$ when $\mu=\mu_{0}$ and decreases steadily as $\mu$ increases when $\mu_{0}<\mu<\pi / 2$.

LEMMA 3. If either (i) $\rho^{\prime}<1 / 2, \mu_{1} \leqq \bar{\mu}_{0}, \mu_{2} \leqq \mu_{0}$ or (ii) $\rho^{\prime} \geqq 1 / 2$, then $-\omega_{2}<\gamma$ $<\omega_{1}$.

We now write

$$
\zeta_{0}=\left(\mu_{0}-\bar{\mu}_{0}\right) / 2 \quad\left(\rho^{\prime}<1 / 2\right), \quad \zeta_{0}=0 \quad\left(\rho^{\prime} \geqq 1 / 2\right) .
$$

In any particular example of the former case the value of $\zeta_{0}$ may be calculated readily from (2.8). We choose arg $x$ and arg $(-x)$ so that

$$
-\pi<\arg ( \pm x)-\tan \gamma \log |x|-\zeta_{0} / \rho^{\prime} \leqq \pi
$$

and write

$$
X=x^{\rho}, \quad X^{\prime}=(-x)^{\rho} e^{-\pi \rho i}, \quad X^{\prime \prime}=(-x)^{\rho} e^{\pi \rho i} .
$$

Since $\arg X=\rho^{\prime}(\arg x-\tan \gamma \log |x|)$, it follows that

$$
-\pi \rho^{\prime}<\arg X-\zeta_{0} \leqq \pi \rho^{\prime}
$$

and $X=X^{\prime}$ or $X^{\prime \prime}$ according as $\arg X \leqq \zeta_{0}$ or $\arg X>\zeta_{0}$. Thus $\arg X$ (but not $\arg x)$ is bounded as $|x| \rightarrow \infty$. If $\rho^{\prime}<1 / 2,(2.9)$ is equivalent to

$$
-\bar{\mu}_{0}<\arg X \leqq \mu_{0}
$$

by (2.7). Finally $Y$ is any large complex number with bounded argument, so that $|Y|>K$ and $|\arg Y|<K$.

3. Statement of results in the first problem. My main result is the following theorem.

THEOREM 1. If $c_{0}(t)$ is regular and satisfies (1.8) in the $\left(-\mu_{1}, \mu_{2}\right)$ sector and if

$$
-\min \left(\mu_{1}, \omega_{2}\right)+\epsilon \leqq \arg X \leqq \min \left(\mu_{2}, \omega_{1}\right)-\epsilon,
$$

then

$$
f(x)=I(X)
$$

where

$$
I(Y)=2^{1 / 2} \pi^{1 / 2} \kappa^{-1-\beta} Y^{1 / 2+\beta} e^{P(Y)}\left\{1+O\left(Y^{-K}\right)\right\} .
$$

When $\arg X$ satisfies (3.1), it follows from (2.5) that $R(X)>K|X|$. Relation (3.2) implies that

$$
\log f(x) \sim P(X) \sim X
$$


so that, in an obvious sense, $f(x)$ is exponentially large. The whole of the $x$-plane is not covered by (3.1) and, if $\rho^{\prime} \geqq 1 / 2$, there may be parts of the plane in which $f(x)$ is not exponentially large and in which the asymptotic behavior of $f(x)$ does not depend solely on that of $c_{0}(t)$ for large $t$. This possibility was sufficiently exemplified in Theorems 5-8 of [11].

When $\rho^{\prime}<1 / 2$, however, we can find an asymptotic formula for $f(x)$ valid for all large $x$, provided $c_{0}(t)$ satisfies the conditions of Theorem 1 in a suitable sector. The result is best expressed in terms of $X^{\prime}, X^{\prime \prime}$ and is as follows.

THEOREM 2. If $\rho^{\prime}<1 / 2$ and if $c_{0}(t)$ is regular and satisfies (1.8) in the $\left(-\bar{\mu}_{0}-\epsilon, \mu_{0}+\epsilon\right)$ sector, then

$$
f(x)=I\left(X^{\prime}\right)+I\left(X^{\prime \prime}\right)
$$

for all large $x$.

The connection between this result and Theorem 1 is made somewhat clearer by the following lemma, which follows from (4.12) and (4.13) of [14].

LEMMA 4. If $\rho^{\prime}<1 / 2$, then

$$
\begin{aligned}
& \mathcal{R}\left(Y-Y e^{-2 \pi i \rho}\right)=K|Y| \sin \left(\mu_{0}-\arg Y\right), \\
& \mathcal{R}\left(Y-Y e^{2 \pi i \rho}\right)=K|Y| \sin \left(\bar{\mu}_{0}+\arg Y\right) .
\end{aligned}
$$

When $\rho^{\prime}<1 / 2, \arg X$ always satisfies (2.10). Except near the ends of this interval $R(X)$ is greater than either of $R\left(X e^{ \pm 2 \pi i \rho}\right)$. Thus, when $-\bar{\mu}_{0}+K$ $\leqq$ arg $X \leqq \zeta_{0}$, for example, $X^{\prime}=X$ and $R\left(X^{\prime}\right)>R\left(X^{\prime \prime}\right)-K\left|X^{\prime}\right|$, so that $I\left(X^{\prime \prime}\right)$ is negligible compared with the error term in $I\left(X^{\prime}\right)$. Hence (3.2) and (3.3) are equivalent. When $\zeta_{0}<\arg X \leqq \mu_{0}-K$, the same is true with $X^{\prime}, X^{\prime \prime}$ interchanged. But, when (3.1) is false and arg $X$ is very near $-\bar{\mu}_{0}$ (say), $R\left(X^{\prime}\right)$ and $R\left(X^{\prime \prime}\right)$ are nearly equal and so both $I\left(X^{\prime}\right)$ and $I\left(X^{\prime \prime}\right)$ are relevant.

It follows from Lemma 4 that (3.3) is equivalent to the statement that

$$
f(x)=\sum_{\left|\arg _{x}^{\rho}\right|<\pi / 2} I\left(x^{\rho}\right),
$$

the extra terms, if any, being negligible compared with the error term in $I\left(X^{\prime}\right)$ or in $I\left(X^{\prime \prime}\right)$, whichever is the larger.

For all $\rho^{\prime}>0$, if we know only that $c_{0}(t)$ satisfies (1.8) for all large integral $t$, without any condition of regularity, we can still deduce something about the behavior of $f(x)$, namely:

TheOREM 3. If (1.8) is true for all large, positive integral values of $t$ and if

$$
\left|\arg X-\gamma+\Im\left\{\sum_{1 \leqq s \leqq c / 2}(-1)^{s-1} \sum_{(s)} T \tau^{s-1} \rho^{s-\tau} X^{-\tau}\right\}\right|<K|X|^{-1 / 2}
$$

then $f(x)=I(X)$. If $b \leqq 1 / 2,(3.4)$ becomes 


$$
|\arg X-\gamma| \leqq K|X|^{-1 / 2} \text {. }
$$

Clearly the conditions of this theorem are satisfied when $x$ is in the neighborhood of a certain curve which behaves in distant parts of the plane rather like the equiangular spiral arg $X=\gamma$. Unless $b \leqq 1 / 2$, however, the conditions of the theorem may not be satisfied at all points actually on this spiral.

As we remarked in $\$ 1$, if we are given a more detailed approximation of $c_{0}(t)$ we can deduce a correspondingly more detailed approximation to $f(x)$. Our result is:

TheOREM 4. If we replace the condition (1.8) in Theorems 1, 2 and 3 by the more precise condition (1.12), we may replace $I(X)$ by the $I_{1}(X)$ of (1.13) and correspondingly for $I\left(X^{\prime}\right)$ and $I\left(X^{\prime \prime}\right)$, where the $\beta_{m}^{\prime}$ and $B_{m}^{\prime}$ of $I_{1}(X)$ can be calculated in terms of the $\beta_{m}$ and the $B_{m}$ of (1.12).

We omit the proof of Theorem 4, since the extensions required to the proof of the other theorems are more tedious than difficult. In $\$ 9$, however, we give a rule to calculate the $\beta_{m}^{\prime}$ and the $B_{m}^{\prime}$.

4. The functions $U(Y)$ and $\phi(U)$. The following lemma is a well known result due to Lagrange; see, for example, [8, p. 133].

Lemma 5. Let $A$ be the circumference of the circle $|z|=K_{1}$ in the $z$-plane, let $p(z)$ and $\vartheta(z)$ be functions of $z$ regular on and within $\mathcal{A}$ and let $|\vartheta(z)|<|z|$ at all points on $\mathcal{A}$. Then the equation $z=\vartheta(z)$ has just one root $z=Z$ within $\mathcal{A}$ and

$$
p(Z)=p(0)+\sum_{s=1}^{\infty} \frac{1}{s !}\left[\frac{d^{s-1}}{d z^{s-1}}\left\{p^{\prime}(z) \vartheta^{s}\right\}\right]_{z=0} .
$$

We take $\vartheta=\vartheta(z)=\rho \psi^{\prime}\left(\rho Y e^{z}\right)=\sum A_{j} b_{j} Y^{b_{j}-1} e^{\left(b_{j}-1\right) z}$, so that

$$
|\vartheta(z)|<K|Y|^{b-1}<K_{1}=|z|
$$

on $\mathcal{A}$, provided $|Y|>K$. Hence $Z$ is uniquely defined as a function of $Y$. We define $U=U(Y)$ and $\phi(U)$ by

$$
U=U(Y)=Y e^{z}, \quad \phi(U)=U+\sum A_{j}\left(1-b_{j}\right) U^{b_{i}} .
$$

By (2.3),

$$
\frac{1}{s !} \vartheta^{s}=\sum_{(s)} T \rho^{8-\tau} Y^{-\tau} e^{-\tau z} .
$$

Putting $p(z)=z$ in Lemma 5 , we have

$$
\frac{1}{s !}\left[\frac{d^{s-1}}{d z^{s-1}}\left\{p^{\prime}(z) \vartheta^{s}\right\}\right]_{z=0}=\sum_{(s)}(-\tau)^{s-1} T \rho^{s-\tau} Y^{-\tau}
$$

and so 


$$
\log \left(\frac{U}{Y}\right)=Z=\sum_{s=1}^{\infty}(-1)^{s-1} \sum_{(s)} T \tau^{s-1} \rho^{\varepsilon-\tau} Y^{-\tau} .
$$

If we put $p(z)=e^{d z}$ for any fixed $d$ we have similarly

$$
U^{d}=Y^{d} e^{d Z}=Y^{d}\left\{1+d \sum_{s=1}^{\infty} \sum_{(s)}(d-\tau)^{s-1} T \rho^{s-\tau} Y^{-\tau}\right\} .
$$

Again the equation $Z=\vartheta(Z)$ is equivalent to

$$
Y=U \exp \left(-\sum A_{j} b_{j} U^{b_{j}-1}\right) .
$$

We next take

$$
p(z)=e^{z}+\sum A_{j}\left(1-b_{j}\right) Y^{b_{j}-1} e^{b_{j} z},
$$

so that

$$
\phi(U)=Y p(Z)
$$

and

$$
p^{\prime}(z)=e^{z}+\sum A_{j} b_{j}\left(1-b_{j}\right) Y^{b_{j}-1} e^{b_{j} z}=e^{z}\left\{1-\vartheta^{\prime}(z)\right\} .
$$

Now

$$
(s+1) e^{z} \vartheta^{\prime} \vartheta^{\bullet}=e^{z} \frac{d}{d z} \vartheta^{\diamond+1}=\frac{d}{d z}\left(e^{z} \vartheta^{\diamond+1}\right)-e^{z} \vartheta^{\diamond+1}
$$

and so

$$
\begin{aligned}
\sum_{s=1}^{\infty} \frac{1}{s !} \frac{d^{s-1}}{d z^{s-1}}\left\{p^{\prime}(z) \vartheta^{s}\right\}= & \sum_{s=1}^{\infty} \frac{1}{s !} \frac{d^{\bullet-1}}{d z^{o-1}}\left(e^{z} \vartheta^{\varepsilon}-e^{z} \vartheta^{\prime} \vartheta^{s}\right) \\
= & \sum_{s=1}^{\infty} \frac{1}{s !} \frac{d^{\bullet-1}}{d z^{s-1}}\left(e^{z} \vartheta^{s}\right)-\sum_{s=1}^{\infty} \frac{1}{(s+1) !} \frac{d^{s}}{d z^{s}}\left(e^{z} \vartheta^{s+1}\right) \\
& +\sum_{s=1}^{\infty} \frac{1}{(s+1) !} \frac{d^{s-1}}{d z^{s-1}}\left(e^{z} \vartheta^{s+1}\right) \\
= & e^{z \vartheta}+\sum_{s=2}^{\infty} \frac{1}{s !} \frac{d^{s-2}}{d z^{s-2}}\left(e^{z} \vartheta^{s}\right) .
\end{aligned}
$$

Also

$$
p(0)+\vartheta(0)=1+\sum A_{j} Y^{b_{j}-1}=1+Y^{-1} \psi(\rho Y) .
$$

The formulae (4.1), (4.6), (4.7) and (4.8) together give us

$$
\phi(U)=Y+\psi(\rho Y)+\sum_{s=2}^{\infty} \sum_{(s)}(1-\tau)^{\rho-2} T \rho^{s-\tau} Y^{1-\tau} .
$$


If we put $d=1$ in (4.4) and compare it with (4.9), we see that

$$
\frac{d}{d Y} \phi(U)=\frac{U}{Y} \text {. }
$$

It follows from (2.4) that the series in (4.3), (4.4) and (4.9) are not only convergent for $|Y|>K$ but also asymptotic in the sense that, if only a finite number of terms are taken, the error is of the order of the term of greatest order neglected. In particular,

$$
\begin{gathered}
U=Y+O\left(Y^{b}\right), \quad \arg U=\arg Y+O\left(Y^{b-1}\right), \\
\phi(U)=Y+O\left(Y^{b}\right) .
\end{gathered}
$$

Again, when $s \geqq[c]+1>c$, (2.4) shows that $R(\tau) \geqq s(1-b)>1$ and so, by (4.9) and the definition of $P(Y)$,

$$
\phi(U)=P(Y)+o(1), \quad e^{\phi(U)} \sim e^{P(Y)} .
$$

5. Use of Cauchy's theorem. We write

$$
\begin{gathered}
u=\kappa t, \quad r=|u|, \quad \theta=\arg u, \\
\chi(u)=\chi(u, Y)=u \log (e Y / u)+\psi(\rho u) .
\end{gathered}
$$

We choose $n_{1}$ a fixed positive integer such that, if $K_{2}=\kappa^{\prime}\left(n_{1}-1 / 2\right), K_{2}$ is greater than the $K$ of $(2.6) ; N>K|Y|$ is a positive integer. The contour $\mathbb{B}$ is the segment of the straight line $R(u)=K_{2}$ on which $-\mu_{1} \leqq \theta \leqq \mu_{2}$. The contour $\mathcal{C}_{N}\left(\theta_{1}\right)$ is the segment of the straight line $\theta=\theta_{1}$ on which $K_{2} \sec \theta_{1} \leqq r$ $\leqq(N+1 / 2)|\kappa|$, while $\mathcal{C}\left(\theta_{1}\right)$ is the semi-infinite straight line on which $\theta=\theta_{1}$ and $r \geqq K_{2} \sec \theta_{1}$. The contour $\mathcal{D}_{N}$ is the arc of the circle $r=(N+1 / 2)|\kappa|$ on which $-\mu_{1} \leqq \theta \leqq \mu_{2}$.

We now suppose $c_{0}(t)$ regular and (1.8) satisfied in the $\left(-\mu_{1}, \mu_{2}\right)$ sector in the $t$-plane and $u$ confined to the corresponding sector in the $u$-plane, so that $-\mu_{1} \leqq \theta \leqq \mu_{2}$ and $\cos \theta>K$. By (1.8) and (5.1),

$$
c_{0}(\rho u) Y^{u} \sim \rho^{\beta} u^{\beta} e^{\boldsymbol{X}(u, Y)}
$$

and

$$
\begin{aligned}
& \log \left|c_{0}(\rho u) Y^{u}\right|=R\{\chi(u, Y)\}+O(\log r) \\
& \quad=r \cos \theta \log (e|Y| / r)+r \sin \theta(\theta-\arg Y)+O\left(r^{b}\right)<-K r,
\end{aligned}
$$

provided $r>K|Y|$. Hence, if we write

$$
H\left(\theta_{1}, Y\right)=\int_{\mathcal{C}_{\left(\theta_{1}\right)}} c_{0}(\rho u) Y^{u} d u, \quad \bar{H}\left(\theta_{1}, Y\right)=\int_{\mathcal{C}_{\left(\theta_{1}\right)}}\left|c_{0}(\rho u) Y^{u}\right| d r,
$$

the integrals are both convergent when $-\mu_{1} \leqq \theta \leqq \mu_{2}$. We now prove the following lemma. 
LEMMA 6. If $c_{0}(t)$ is regular and (1.8) holds in the $\left(-\mu_{1}, \mu_{2}\right)$ sector and if $-\mu_{1} \leqq \theta_{1}, \theta_{2} \leqq \mu_{2}$, then

$$
H\left(\theta_{1}, Y\right)=H\left(\theta_{2}, Y\right)+O\left(Y^{K}\right)
$$

On $\mathcal{D}_{N}$

$$
\left|c_{0}(\rho u) Y^{u}\right|<K e^{-K N}
$$

by (5.3), while

$$
\left|c_{0}(\rho u) Y^{u}\right|<K|Y| K
$$

on $B$. The function $c_{0}(\rho u) Y^{u}$ is regular on and within the contour formed by $\mathcal{C}_{N}\left(\theta_{1}\right), \mathcal{C}_{N}\left(\theta_{2}\right)$ and the portions of $\mathcal{B}$ and $\mathcal{D}_{N}$ joining their extremities. Hence, by Cauchy's theorem,

$$
\int_{\mathcal{C}_{N\left(\theta_{1}\right)}}-\int_{\mathcal{C}_{N\left(\theta_{2}\right)}} c_{0}(\rho u) Y^{u} d u=O\left(Y^{K}\right)+O\left(N e^{-K N}\right) .
$$

If we let $N \rightarrow \infty$, Lemma 6 follows.

LEMMA 7. If $c_{0}(t)$ is regular and (1.8) holds in the $\left(-\mu_{1}, \mu_{2}\right)$ sector, then

$$
\begin{aligned}
& f\left(Y^{\star}\right)=O\left\{\bar{H}\left(-\mu_{1}, Y\right)\right\}+O\left\{\bar{H}\left(\mu_{2}, Y e^{2 \pi i \rho}\right)\right\}+O\left(Y^{K}\right), \\
& \kappa f\left(Y^{\star}\right)=H(\gamma, Y)+O\left\{\bar{H}\left(-\mu_{1}, Y e^{-2 \pi i \rho}\right)\right\}+O\left\{\bar{H}\left(\mu_{2}, Y e^{2 \pi i \rho}\right)\right\}+O\left(Y^{K}\right),
\end{aligned}
$$

and

$$
\begin{aligned}
\kappa f\left(Y^{\kappa}\right)= & H(\gamma, Y)+H\left(\gamma, Y e^{-2 \pi i \rho}\right)+O\left\{\bar{H}\left(-\mu_{1}, Y e^{-4 \pi i \rho}\right)\right\} \\
& +O\left\{\bar{H}\left(\mu_{2}, Y e^{2 \pi i \rho}\right)\right\}+O\left(Y^{K}\right) .
\end{aligned}
$$

We integrate the function $c_{0}(\rho u) Y^{u}\left(1-e^{-2 \pi i \rho u}\right)^{-1}$ round the closed contour formed by $\mathcal{B}, \mathcal{C}_{N}\left(-\mu_{1}\right), \mathcal{D}_{N}$ and $\mathcal{C}_{N}\left(\mu_{2}\right)$. The integrand is regular on and within this contour except for a simple pole at each of the points $u=\kappa n\left(n_{1} \leqq n \leqq N\right)$, where $2 \pi i$ times the residue is $\kappa c_{0}(n) Y^{\star n}$. On $\mathbb{B}$ and on $\mathcal{D}_{N}$,

$$
\left|1-e^{-2 \pi i \rho u}\right|>K \text {. }
$$

Combining this with (5.4) and (5.5) and letting $N \rightarrow \infty$, we have

$$
\kappa f\left(Y^{\star}\right)=\int_{\mathcal{C}_{\left(-\mu_{1}\right)}}-\int_{\mathcal{C}_{\left(\mu_{2}\right)}} c_{0}(\rho u) Y^{u}\left(1-e^{-2 \pi i \rho u}\right)^{-1} d u+O\left(Y^{K}\right)
$$

by Cauchy's theorem.

Now

$$
\begin{aligned}
\left(1-e^{-2 \pi i \rho u}\right)^{-1} & =1+e^{-2 \pi i \rho u}\left(1-e^{-2 \pi i \rho u}\right)^{-1} \\
& =1+e^{-2 \pi i \rho u}+e^{-4 \pi i \rho u}\left(1-e^{-2 \pi i \rho u}\right)^{-1} .
\end{aligned}
$$

Since (5.9) holds also on $C\left(-\mu_{1}\right)$ by (2.5), we see that the first integral in 
(5.10) may be replaced by any one of

$$
O\left\{\bar{H}\left(-\mu_{1}, Y\right)\right\}, \quad H\left(-\mu_{1}, Y\right)+O\left\{\bar{H}\left(-\mu_{1}, Y e^{-2 \pi i \rho}\right)\right\},
$$

or

$$
H\left(-\mu_{1}, Y\right)+H\left(-\mu_{1}, Y e^{-2 \pi i \rho}\right)+O\left\{\bar{H}\left(-\mu_{1}, Y e^{-4 \pi i \rho}\right)\right\} .
$$

Similarly $\left(1-e^{-2 \pi i \rho u}\right)^{-1}=e^{2 \pi i \rho u}\left(e^{2 \pi i \rho u}-1\right)^{-1}$ and $\left|e^{2 \pi i \rho u}-1\right|>K$ on $\mathcal{C}\left(\mu_{2}\right)$, so that the second integral in (5.10) is $O\left\{\bar{H}\left(\mu_{2}, Y e^{2 \pi i \rho}\right)\right\}$. Since $-\mu_{1}<\gamma<\mu_{2}$, Lemma 6 is applicable and the three results of Lemma 7 follow at once.

6. Evaluation of $H(\gamma, Y)$.

LEMMA 8. If

$$
-\mu_{1} \leqq \arg U(Y) \leqq \mu_{2}
$$

and if $c_{0}(t)$ is regular and satisfies (1.8) in the $\left(-\mu_{1}, \mu_{2}\right)$ sector, then

$$
H(\gamma, Y) \sim 2^{1 / 2} \pi^{1 / 2} \kappa^{-\beta} U^{1 / 2+\beta} e^{\phi(U)}
$$

and

$$
H(\gamma, Y)=\kappa I(Y) .
$$

By (4.11), (4.13) and the definition of $I(Y)$ in Theorem 1, (6.2) and (6.3) are equivalent; we shall prove (6.2). Throughout this section we suppose (6.1) to hold good; hence, by Lemma 6,

$$
H(\gamma, Y)=H(\arg U, Y)+O\left(Y^{K}\right) .
$$

Also cos $\arg U>K$ by (2.5) and so

$$
R\{\phi(U)\} \sim R(U)>K|U|>K|Y|
$$

by (4.11) and (4.12). By (5.2),

$$
H(\arg U, Y)=\rho^{\beta} \int_{\mathcal{C}_{(\arg U)}} u^{\beta} e^{\chi(u)}\left\{1+O\left(u^{-K}\right)\right\} d u .
$$

By (4.2), (4.5) and (5.1), we see that

$$
\begin{gathered}
\chi(U)=\phi(U), \\
\chi^{\prime}(u)=\log (Y / u)+\rho \psi^{\prime}(\rho u), \quad \chi^{\prime}(U)=0,
\end{gathered}
$$

so that $u=U$ is a "saddle-point" for the integral (6.6). Also

$$
\chi^{\prime \prime}(u)=-\frac{1}{u}+O\left(u^{b-2}\right)
$$

for large $u$. In the integral (6.6) we put $u=U(1+v)$, so that $v$ is real, and write 


$$
L(v)=\chi(u)-\chi(U) .
$$

Hence $L(0)=L^{\prime}(0)=0$ and, by $(6.8)$,

$$
L^{\prime \prime}(v)=U^{2} \chi^{\prime \prime}(u)=-\frac{U}{1+v}\left\{1+O\left(u^{b-1}\right)\right\} .
$$

Hence, if $|u|$ is greater than some $K_{3}$,

$$
R\left\{L^{\prime \prime}(v)\right\}<-\frac{K R(U)}{1+v}<-\frac{K|U|}{1+v}
$$

by (6.5).

We now choose $\epsilon$ so that $0<\epsilon<\min \{1 / 6,(1-b) / 2\}$, write $k=|U|^{-1 / 2}$, divide $\mathcal{C}(\arg U)$ into four parts, viz. those on which

$$
\begin{aligned}
K_{2} \sec \arg U & \leqq|u| \leqq K_{3} ; \\
K_{3}<|u| & <|U|(1-k), \text { that is, } v<-k ; \\
(1-k)|U| & \leqq|u| \leqq|U|(1+k), \text { that is, }-k \leqq v \leqq k ; \\
(1+k)|U| & <|u|, \text { that is, } v>k
\end{aligned}
$$

respectively and write

$$
H(\arg U, Y)=H_{1}+H_{2}+H_{3}+H_{4}
$$

to correspond. We have at once

$$
\left|H_{1}\right|<K|Y| K \text {. }
$$

In $H_{2}, H_{3}$, and $H_{4},|u|>K_{3}$ and so (6.9) is true. By Taylor's theorem for a real function of a real variable,

$$
\begin{aligned}
R\{L(v)\} & =R\{L(0)\}+v R\left\{L^{\prime}(0)\right\}+\left(v^{2} / 2\right) R\left\{L^{\prime \prime}(w v)\right\} \\
& =\left(v^{2} / 2\right) R\left\{L^{\prime \prime}(w v)\right\}
\end{aligned}
$$

for some $w$ such that $0<w<1$. By (6.9),

$$
R\left\{L^{\prime \prime}(w v)\right\}<-\frac{K|U|}{1+w v}< \begin{cases}-K|U| & (v<0), \\ -\frac{K|U|}{1+v} & (v>0) .\end{cases}
$$

Hence, when $v \leqq-k$,

$$
R\{L(v)\}<-K v^{2}|U| \leqq-K|U|^{2 *}
$$

and so

$$
\left|H_{2} e^{-x(U)}\right| \leqq K|U| K^{-K|U| 2 e} \leqq K\left|U^{1 / 2+\beta-K}\right|
$$

for some $K$. Again 


$$
\begin{aligned}
\left|H_{4} e^{-\chi(U)}\right| & \leqq K\left|U^{K} \int_{k}^{\infty}(1+v)^{\beta} e^{L(v)} d v\right| \\
& \leqq K|U| K \int_{k}^{\infty}\left|(1+v)^{\beta}\right| e^{-K|U| v^{2} /(1+v)} d v \\
& \leqq K|U| K\left(\int_{k}^{1} e^{-K|U| v 2} d v+\int_{1}^{\infty}\left|(1+v)^{\beta}\right| e^{-K|U| v} d v\right) \\
& \leqq K|U| K e^{-K|U|^{2 \epsilon}} \leqq K\left|U^{1 / 2+\beta-K}\right| .
\end{aligned}
$$

To calculate $H_{3}$ we require the following well known result.

LEMMA 9. If $0<\epsilon<1 / 2, h>K|y|^{-1 / 2}$ and $|\arg y|<\pi / 2-K$, then

$$
\int_{-h}^{h} e^{-y v^{2} / 2} d v \sim\left(\frac{2 \pi}{y}\right)^{1 / 2}, \quad \int_{-h}^{h}\left|e^{-y v^{2} / 2}\right| d v=O\left(\frac{1}{y^{1 / 2}}\right)
$$

as $y \rightarrow \infty$.

It follows from (4.5) and the definition of $v$ and $L(v)$ that

$$
L(v)=U\{v-(1+v) \log (1+v)\}+\sum A_{j} U^{b_{i}}\left\{(1+v)^{b_{j}}-1-b_{j} v\right\} .
$$

Hence, when $|v| \leqq k \equiv|U|^{\bullet-1 / 2}$, we have

$$
\begin{aligned}
\left|L(v)+U v^{2} / 2\right| & \leqq K\left|U v^{3}\right|+K\left|U^{b} v^{2}\right| \\
& \leqq K\left(|U|^{3 \epsilon-1 / 2}+|U|^{2 \epsilon+b-1}\right) \leqq K|U|^{-K},
\end{aligned}
$$

since $\epsilon<\min \{1 / 6,(1-b) / 2\}$, and so

$$
H_{3} e^{-\chi(U)}=\kappa^{-\beta} U^{\beta+1} \int_{-k}^{k} e^{-U v^{2} / 2}\left\{1+O\left(U^{-K}\right)\right\} d v \sim 2^{1 / 2} \pi^{1 / 2} \kappa^{-\beta} U^{\beta+1 / 2}
$$

by Lemma 9. (6.2) follows from (6.4), (6.5), (6.7) and (6.10)-(6.14). lemma.

We observe that our method would enable us to prove the following

LEMMA 10. If $|\arg U(Y)|<\pi / 2-K$, then

$$
\int_{\mathcal{C}_{(\arg U)}}\left|u^{\beta} e^{X(u, Y)}\right||d u|<K\left|U^{1 / 2+\beta} e^{\phi(u)}\right|
$$

If we put $J=1, A_{1}=K, b_{1}=b$ in this lemma and replace $Y$ by $|Y| \sigma$, where $K<\sigma<K$, then $U(|Y| \sigma)$ is real and arg $U=0$. Writing $R$ for the real variable $u$ and $\beta=R(\beta)$, we have:

LEMMA 11. If $K<\sigma<K$ and $Y$ is any large number, then

$$
\log \int_{K}^{\infty} R^{R\left(\beta^{\prime}\right)} \exp \left\{R \log \left(\frac{e|Y| \sigma}{R}\right)+K R^{b}\right\} d R \leqq|Y| \sigma+K|Y|^{1-K} \text {. }
$$


7. Proof of Theorems 1 and 2. Our next step is to find an upper bound for the $\bar{H}$ of Lemma 7 .

LEMMA 12. If $\cos \theta>K$ and if (1.8) is true on $\mathcal{C}(\theta)$, then.

$$
\log \bar{H}(\theta, Y) \leqq|Y| \cos \theta e^{\tan \theta(\theta-\arg Y)}+K|Y|^{1-K} .
$$

By (1.8)

$$
\bar{H}(\theta, Y)<K \int_{K_{2} \sec \theta}^{\infty} r^{m\left(\beta^{\prime}\right)} e^{\nu_{1}} d r
$$

where

$$
\nu_{1}=r \cos \theta \log (e|Y| / r)+r \sin \theta(\theta-\arg Y)+O\left(r^{b}\right) .
$$

If we put $R=r \cos \theta$ and $\sigma=\cos \theta e^{\tan \theta(\theta-\arg Y)}$ in Lemma 11, the conditions of that lemma are satisfied and Lemma 12 follows at once.

Replacing $Y$ by $Y e^{-2 \pi i \rho}$ in Lemma 12, we have

$$
\log \bar{H}\left(-\mu_{1}, Y e^{-2 \pi i \rho}\right) \leqq|Y| \lambda_{1}(\arg Y)+K|Y|^{1-K},
$$

where

$$
\lambda_{1}(\eta)=\cos \mu_{1} \exp \left\{\left(\mu_{1}+\eta-2 \pi \rho^{\prime}\right) \tan \mu_{1}-2 \pi \rho^{\prime} \tan \gamma\right\} .
$$

Similarly

$$
\log \bar{H}\left(\mu_{2}, Y e^{2 \pi i \rho}\right) \leqq|Y| \lambda_{2}(\arg Y)+K|Y|^{1-K}
$$

where

$$
\lambda_{2}(\eta)=\cos \mu_{2} \exp \left\{\left(\mu_{2}-\eta-2 \pi \rho^{\prime}\right) \tan \mu_{2}+2 \pi \rho^{\prime} \tan \gamma\right\} .
$$

LEMMA 13. (i) $\lambda_{1}(\eta) \leqq \cos \eta$ if $-\mu_{1} \leqq \eta \leqq \omega_{1}$.

(ii) $\lambda_{1}(\eta) \leqq \cos \eta-K$ if $-\mu_{1} \leqq \eta \leqq \omega_{1}-\epsilon$.

(iii) $\lambda_{2}(\eta) \leqq \cos \eta$ if $-\omega_{2} \leqq \eta \leqq \mu_{2}$.

(iv) $\lambda_{2}(\eta) \leqq \cos \eta-K$ if $-\omega_{2}+\epsilon \leqq \eta \leqq \mu_{2}$.

Let $-\omega_{2} \leqq \eta \leqq \mu_{2}$, so that $\cos \eta>K$, and let $\zeta(\eta)=\cos \eta-\lambda_{2}(\eta)$. We have $\zeta\left(-\omega_{2}\right)=0$ by the definition of $\omega_{2}$ and

$$
\zeta\left(\mu_{2}\right)=\cos \mu_{2}\left(1-\exp \left\{2 \pi \rho^{\prime}\left(\tan \gamma-\tan \mu_{2}\right)\right\}\right)>K,
$$

since $\gamma<\mu_{2}$. But

$$
\zeta^{\prime \prime}(\eta)=-\cos \eta-\tan ^{2} \mu_{2} \lambda_{2}(\eta)<-K
$$

when $-\omega_{2} \leqq \eta \leqq \mu_{2}$ and so

$$
\zeta(\eta) \geqq 0 \quad\left(-\omega_{2} \leqq \eta \leqq \mu_{2}\right), \quad \zeta(\eta)>K\left(-\omega_{2}+\epsilon \leqq \eta \leqq \mu_{2}\right),
$$

which are (iii) and (iv). The proof of (i) and (ii) is similar.

Now suppose the conditions of Theorem 1 satisfied, so that 


$$
-\min \left(\mu_{1}, \omega_{2}\right)+\epsilon \leqq \arg X \leqq \min \left(\mu_{2}, \omega_{1}\right)-\epsilon
$$

by (3.1). Hence, by (7.1), (7.2) and Lemma 13 (ii) and (iv),

$$
\bar{H}\left(-\mu_{1}, X e^{-2 \pi i \rho}\right)+\bar{H}\left(\mu_{2}, X e^{2 \pi i \rho}\right)=O\left(e^{X-K|X|}\right) .
$$

Hence, by (5.7),

$$
\kappa f(x)=H(\gamma, X)+O\left(e^{X-K|x|}\right)+O\left(X^{K}\right) .
$$

Since $\arg U(X) \sim \arg X$, it follows from (7.3) that (6.1) is satisfied by $U(X)$ and so, by Lemma 8 ,

$$
f(x)=I(X)+O\left(e^{X-K|X|}\right)+O\left(X^{K}\right) .
$$

Now $|\arg X|<\pi / 2-K$ by (7.3) and (2.5) and so $R\{P(X)\} \sim R(X)>K|X|$. Hence the error terms in (7.4) can be absorbed in that of $I(X)$ and

$$
f(x)=I(X),
$$

which is Theorem 1.

We now suppose the conditions of Theorem 2 satisfied so that, in particular, $\rho^{\prime}<1 / 2$. Since $\epsilon$ is any sufficiently small positive number, we may replace $\epsilon$ by $2 \epsilon$ in our hypotheses, so that $c_{0}(t)$ is regular and (1.8) is true in the $\left(-\bar{\mu}_{0}-2 \epsilon, \mu_{0}+2 \epsilon\right)$ sector. We take $\epsilon$ small enough to ensure that

$$
\max \left(\bar{\mu}_{0}, \mu_{0}\right)+2 \epsilon<\pi / 2, \quad \epsilon<\left(\mu_{0}+\bar{\mu}_{0}\right) / 2,
$$

the latter being possible by (2.7). We shall prove Theorem 2 when

$$
0<\arg x-\tan \gamma \log |x|-\left(\mu_{0}-\bar{\mu}_{0}\right) /\left(2 \rho^{\prime}\right) \leqq \pi,
$$

the proof when

$$
-\pi<\arg x-\tan \gamma \log |x|-\left(\mu_{0}-\bar{\mu}_{0}\right) /\left(2 \rho^{\prime}\right) \leqq 0
$$

being similar. It follows from (7.7) that

$$
-\pi<\arg (-x)-\tan \gamma \log |x|-\left(\mu_{0}-\bar{\mu}_{0}\right) /\left(2 \rho^{\prime}\right) \leqq 0
$$

and so that $X^{\prime \prime}=X, X^{\prime}=X e^{-2 \pi i p}$ and

$$
\left(\mu_{0}-\bar{\mu}_{0}\right) / 2<\arg X=\arg X^{\prime} \leqq \mu_{0} .
$$

We consider separately the case in which

$$
\left(\mu_{0}-\bar{\mu}_{0}\right) / 2<\arg X^{\prime \prime} \leqq \mu_{0}-\epsilon
$$

and that in which

$$
\mu_{0}-\epsilon<\arg X^{\prime \prime} \leqq \mu_{0} .
$$

First let (7.9) be true, so that

$$
-\bar{\mu}_{0}+\epsilon<\arg X \leqq \mu_{0}-\epsilon
$$


by (7.6). By Lemma 2(ii), if we put $\mu_{1}=\bar{\mu}_{0}$ and $\mu_{2}=\mu_{0}$, we have $\omega_{1}=\mu_{0}$ and $\omega_{2}=\bar{\mu}_{0}$. Hence the conditions of Theorem 1 are satisfied and $f(x)=I(X)$ $=I\left(X^{\prime \prime}\right)$. But, by Lemma 4 and (7.9),

$$
R\left(X^{\prime \prime}-X^{\prime}\right)=K\left|X^{\prime \prime}\right| \sin \left(\mu_{0}-\arg X^{\prime \prime}\right)>K\left|X^{\prime \prime}\right| \text {. }
$$

Hence $I\left(X^{\prime}\right)$ is negligible compared with the error term in $I\left(X^{\prime \prime}\right)$ and

$$
f(x)=I\left(X^{\prime}\right)+I\left(X^{\prime \prime}\right)
$$

the result of Theorem 2 .

Next let (7.10) be true. Since

$$
\arg X^{\prime}=\arg X^{\prime \prime}-2 \pi \rho^{\prime}=\arg X^{\prime \prime}-\mu_{0}-\bar{\mu}_{0}
$$

by (2.7), we have

$$
-\bar{\mu}_{0}-\epsilon<\arg X^{\prime} \leqq-\bar{\mu}_{0}
$$

By (4.11)

$$
\begin{gathered}
\mu_{0}-2 \epsilon<\arg U\left(X^{\prime \prime}\right)<\mu_{0}+\epsilon, \\
-\bar{\mu}_{0}-2 \epsilon<\arg U\left(X^{\prime}\right)<-\bar{\mu}_{0}+\epsilon .
\end{gathered}
$$

If we take $\mu_{1}=\bar{\mu}_{0}+2 \epsilon$ and $\mu_{2}=\mu_{0}+2 \epsilon$ in Lemma $8,(6.1)$ is satisfied by $U\left(X^{\prime \prime}\right)$ and by $U\left(X^{\prime}\right)$ and so

$$
H\left(\gamma, X^{\prime \prime}\right)=\kappa I\left(X^{\prime \prime}\right), \quad H\left(\gamma, X^{\prime}\right)=\kappa I\left(X^{\prime}\right) .
$$

We now take $\mu_{1}=\bar{\mu}_{0}$ and $\mu_{2}=\mu_{0} ;$ (7.11) will follow at once from (5.8) provided that

$$
X^{\prime \prime K}, \quad \bar{H}\left(-\bar{\mu}_{0}, X^{\prime \prime} e^{-4 \pi i \rho}\right), \quad \bar{H}\left(\mu_{0}, X^{\prime \prime} e^{2 \pi i \rho}\right)
$$

are all $O\left\{\exp \left(X^{\prime \prime}-K\left|X^{\prime \prime}\right|\right)\right\}$ and so negligible compared with the error term in $I\left(X^{\prime \prime}\right)$. Since cos arg $X^{\prime \prime}>K, X^{\prime \prime K}$ is certainly of the required order. Next, putting $Y=X e^{\prime \prime-2 x i \rho}$ in (7.1), we have

$\log \bar{H}\left(-\mu_{1}, X^{\prime \prime} e^{-4 \pi i \rho}\right)$

$$
\begin{aligned}
& \leqq\left|X^{\prime \prime}\right| \lambda_{1}\left(\arg X^{\prime \prime}\right) \exp \left\{-2 \pi \rho^{\prime}\left(\tan \mu_{1}+\tan \gamma\right)\right\}+K\left|X^{\prime \prime}\right| 1-K \\
& \leqq(1-K) \quad\left|X^{\prime \prime}\right| \cos \arg X^{\prime \prime}
\end{aligned}
$$

by Lemmas 13(i) and by (2.5). Finally

$$
\log \bar{H}\left(\mu_{2}, X^{\prime \prime} e^{2 \pi i \rho}\right) \leqq\left|X^{\prime \prime}\right| \lambda_{2}\left(\arg X^{\prime \prime}\right)+K|X| 1-K \leqq R(X)-K|X|
$$

by (7.2) and Lemma 13 (iv), since

$$
-\omega_{2}+\epsilon=-\bar{\mu}_{0}+\epsilon \leqq \arg X^{\prime \prime} \leqq \mu_{0}=\mu_{2} .
$$

8. Proof of Theorem 3. We prove first the following lemma.

LEMMA 14. If 


$$
c_{0}(n)=O\left\{n^{\beta} e^{\psi(n)}(e / \kappa n)^{\kappa n}\right\}
$$

for all integral $n \geqq 1$ and if

$$
\arg U(X)=\gamma+O\left(X^{-1 / 2}\right)
$$

then

$$
f(x)=O\left(X^{1 / 2+\beta} e^{P(x)}\right) .
$$

Let $V$ be real and positive and let

$$
x^{\prime}=(\kappa V)^{\kappa} e^{-\psi^{\prime}(V)}=(\kappa V)^{\kappa} \exp \left(-\sum a_{j} b_{j} V^{b_{j}-1}\right) .
$$

For large $V,\left|x^{\prime}\right|$ is a continuous function of $V$ which tends to infinity with $V$. Hence we can always choose $V$ so that $\left|x^{\prime}\right|=|x|$. Let $t$ be real and positive and let

$$
\Phi(t)=\left|t^{\beta} e^{\psi(t)}(e / \kappa t)^{\kappa t} x^{\prime t}\right|=\left|t^{\beta} e^{\gamma(t)}\right|
$$

where

$$
\nu(t)=\kappa^{\prime} t \log (e V / t)+R\left\{\psi(t)-t \psi^{\prime}(V)\right\}
$$

Hence

$$
\begin{gathered}
\nu^{\prime}(t)=\kappa^{\prime} \log (V / t)+R\left\{\psi^{\prime}(t)-\psi^{\prime}(V)\right\}, \quad \nu^{\prime}(V)=0, \\
\nu^{\prime \prime}(t)=-\frac{\kappa^{\prime}}{t}+R\left\{\psi^{\prime \prime}(t)\right\}=-\frac{\kappa^{\prime}}{t}+O\left(t^{b-2}\right)<-\frac{K}{t}
\end{gathered}
$$

for $t>K$ and we have

$$
\sum_{n>K} \Phi(n) \leqq \max _{n>K} \Phi(n)+\int_{K}^{\infty} \Phi(t) d t \leqq \Phi(V)+\int_{K}^{\infty} t^{R(\beta)} e^{p(t)} d t .
$$

If we put $u=\kappa t, U^{\prime}=\kappa V$ and define $Y^{\prime}$ in terms of $U^{\prime}$ by means of (4.5) we see that $Y^{\prime k}=x^{\prime},\left|Y^{\prime} \alpha\right|=\left|x^{\prime}\right|=|x|$,

$$
\left|\arg U^{\prime}\right|=|\gamma|<\pi / 2-K, \quad \nu(t)=R\left\{\chi\left(u, Y^{\prime}\right)\right\}
$$

by (5.1) and so

$$
\int_{K}^{\infty} t^{R(\beta)} e^{\nu(t)} d t \leqq K+\int_{\mathcal{C}_{(\gamma)}}\left|u^{\beta} e^{\chi\left(u, Y^{\prime}\right)}\right||d u|<K\left|Y^{\prime 1 / 2+\beta} e^{\phi\left(U^{\prime}\right)}\right|
$$

by Lemma 10 and (4.11). Hence

$$
\begin{aligned}
|f(x)| & <K|x|^{K}+\sum_{n>K} \Phi(n) \\
& \leqq \Phi(V)+K\left|Y^{\prime 1 / 2+\beta} e^{\phi\left(U^{\prime}\right)}\right|<K\left|X^{1 / 2+\beta} e^{\phi\left(U^{\prime \prime}\right)}\right|,
\end{aligned}
$$

since $\nu(V)=R\left\{\phi\left(U^{\prime}\right)\right\}$ and $\Phi(V)=\left|V^{\beta}\right| e^{\nu(V)} \sim\left|Y^{\prime \beta} e^{\phi\left(U^{\prime}\right)}\right|$. By (4.13), we have 
only to show that

$$
R\left\{\phi\left(U^{\prime}\right)-\phi(U)\right\}=O(1),
$$

where $U=U(X)$, to complete the proof of Lemma 14 .

Since $|x|=\left|x^{\prime}\right|$, there is a real number $\xi$ such that $x=x^{\prime} e^{i \xi}$ and $X=Y^{\prime} e^{i p \xi}$. Since $\arg X$ and arg $Y^{\prime}$ are bounded, $\xi=O(1)$. Hence

$$
X^{-\tau}-Y^{\prime-\tau}=X^{-\tau}\left(1-e^{-\tau \rho \xi i}\right)=O\left(X^{-\tau} \tau \xi\right)
$$

and so, by (4.3),

$$
\arg U-\gamma=\arg \left(\frac{U}{U^{\prime}}\right)=\arg \left(\frac{X}{Y^{\prime}}\right)+O\left(X^{b-1} \xi\right) \sim \rho^{\prime} \xi .
$$

Let $\Delta(\xi)=R\{\phi(U)\}$, so that, by (4.10),

$$
\begin{aligned}
\Delta^{\prime}(\xi) & =R\left\{\frac{d}{d \xi} \phi(U)\right\}=R\left(\frac{d X}{d \xi} \frac{d \phi(U)}{d X}\right)=R(i \rho U) \\
& =-|\rho U| \sin (\arg U-\gamma)=O\left(\left|Y^{\prime}\right| \xi\right)
\end{aligned}
$$

by (8.2). Hence, by Taylor's theorem, for some $w$ such that $0<w<1$,

$$
R\left\{\phi(U)-\phi\left(U^{\prime}\right)\right\}=\xi \Delta^{\prime}(w \xi)=O\left(\left|Y^{\prime}\right| \xi^{2}\right)=O(1)
$$

by (8.1) and (8.2).

To complete the proof of Theorem 3 we first observe that (3.4) and (8.1) are equivalent by (4.3) and (2.4). We write

$$
\begin{aligned}
c_{0}^{(1)}(n) & =n^{\beta} e^{\psi(n)}(e / \kappa n)^{k n}, & & c_{0}^{(2)}(n)=c_{0}(n)-c_{0}^{(1)}(n), \\
f^{(1)}(x) & =\sum_{n=1}^{\infty} c_{0}^{(1)}(n) x^{n}, & f^{(2)}(x) & =f(x)-f^{(1)}(x)
\end{aligned}
$$

and apply Theorem 1 to $f^{(1)}(x)$ and Lemma 14 to $f^{(2)}(x)$, the latter with $\beta-K$ replacing $\beta$. Theorem 3 follows at once.

9. Rule to calculate $I_{1}(X)$. We now give a rule to calculate the indices and coefficients in $I_{1}(X)$ in Theorem 4. In the following the symbol $v$ (which eventually disappears) is $O(1)$ or, more exactly, satisfies $K<|v|<K$. We suppose that $R\left(\beta_{1}\right) \geqq R\left(\beta_{2}\right) \geqq \cdots \geqq R\left(\beta_{M}\right)>R\left(\beta_{M+1}\right)$. The rule is:

(i) Expand $L\left(U^{-1 / 2} v\right)+v^{2} / 2$ in powers of $v$, reject all terms of order $O\left(U^{\beta_{M+1}-\beta_{1}}\right)$ and denote the resulting polynomial in $v$ by $q$.

(ii) Let $l_{1}$ be the least integer such that $l_{1}>R\left(\beta_{1}-\beta_{M+1}\right) \max (2, c)$, expand

$$
1+q+\frac{q^{2}}{2 !}+\cdots+\frac{q^{l_{1}-1}}{\left(l_{1}-1\right) !}
$$

in powers of $v$ and reject all terms of order $O\left(U^{\beta_{M+1}-\beta_{1}}\right)$. 
(iii) Expand

$$
\sum_{m=1}^{M} B_{m} \kappa^{-\beta_{m}} U^{\beta_{m}}\left(1+U^{-1 / 2} v\right)^{\beta_{m}}
$$

in ascending powers of $v$ and reject all terms of order $O\left(U^{\beta_{M}+1}\right)$.

(iv) Multiply together the polynomials in $v$ obtained in (ii) and (iii), rejecting in the resulting product all terms which are $O\left(U^{\beta_{M+1}}\right)$ and all terms containing odd powers of $v$.

(v) Replace every $v^{2 l}$ in this result by $2^{l} \Gamma(l+1 / 2) / \Gamma(1 / 2)$ and multiply the whole by $\rho 2^{1 / 2} \Gamma(1 / 2) U^{1 / 2}$.

(vi) Replace every $U^{d}$ in the result by its expansion in terms of $Y$ from (4.4), put $Y=X$ and reject all terms of order $O\left(X^{1 / 2+\beta M+1}\right)$.

(vii) Expand

$$
\exp \left\{\sum_{s=[c]+1}^{\infty} \sum_{(s)}(1-\tau)^{s-2} T \rho^{s-\tau} X^{1-\tau}\right\}
$$

in descending powers of $X$, rejecting all terms which are $O\left(X^{1 / 2+\beta M+1}\right)$.

(viii) Multiply the results of (vi) and (vii) together, rejecting all terms of order $O\left(X^{\beta_{M+1}+1 / 2}\right)$ and call the result $\sum_{m=1}^{M^{\prime}} B_{m}^{\prime} X_{m}^{\beta_{m}^{\prime}}$. Then

$$
I_{1}(X)=e^{P(X)}\left\{\sum_{m=1}^{M^{\prime}} B_{m}^{\prime} X_{m}^{\beta_{m}^{\prime}}+O\left(X^{\beta_{M+1}+1 / 2}\right)\right\} .
$$

Unless the difference $R\left(\beta_{1}-\beta_{M+1}\right)$ is fairly small, the above process is as laborious as it appears.

10. The second problem. We now turn our attention to the problem of deducing the asymptotic expansion for large $n$ of $c_{\lambda}(n)$, defined by (1.2), from that of $c_{0}(\boldsymbol{n})$. We write

$$
\begin{aligned}
S_{m} & =\left[\frac{1-m \kappa^{\prime}}{1-b}\right], \\
\psi_{1}(n) & =\kappa^{-\kappa}\left(1+\sum_{s=1}^{S_{1}} \sum_{(s)} T n^{-\tau}\right), \\
\psi_{m}(n) & =m^{-2} \kappa^{-m \kappa}\left\{\left(\begin{array}{c}
m-m \kappa \\
m-1
\end{array}\right)+\sum_{s=1}^{S_{m}} m^{s} \sum_{(s)}\left(\begin{array}{c}
m-m \kappa-\tau \\
m-1
\end{array}\right) T n^{-\tau}\right\}
\end{aligned}
$$

for $m \geqq 2$ and finally

$$
\psi(n, \lambda)=\sum_{m=1}^{\left[1 / \kappa^{\prime}\right]} n^{1-m \kappa} \psi_{m}(n) \lambda^{m-1} .
$$

In each case, if $S_{m}=0$ or $\left[1 / \kappa^{\prime}\right]=0$, the corresponding sum is empty. The main result we shall prove is: 
THEOREM 5. If $c_{0}(t)$ is regular and satisfies (1.8) in some $\left(-\mu_{1}, \mu_{2}\right)$ sector, then

$$
c_{\lambda}(n) \sim c_{0}(n) e^{\lambda \psi(n, \lambda)} \sim n^{\beta} e^{\psi(n)+\lambda \psi(n, \lambda)}(e / \kappa n)^{\kappa n} .
$$

Theorem 5 is almost trivial when $\kappa^{\prime}>1$ but much deeper when $\kappa^{\prime}<1$. Our hypotheses are excessive for $\kappa^{\prime} \geqq 1$ and we have the following theorem:

THEOREM 6. If $\kappa^{\prime} \geqq 1$ and if (1.8) is true for all integral values of $t \geqq n$ then (10.1) is true, where $\psi(n, \lambda)=0\left(\kappa^{\prime}>1\right), \psi(n, \lambda)=\kappa^{-\kappa} n^{1-\kappa}\left(\kappa^{\prime}=1\right)$.

It is natural to enquire whether the restriction $\kappa^{\prime} \geqq 1$ arises from some ineffectiveness in our methods or from the nature of the results. The answer is given in the following theorem.

THEOREM 7. The condition $\kappa^{\prime} \geqq 1$ in Theorem 6 is necessary.

We remark that, if (1.8) is replaced by (1.12) in Theorem 5 , we can replace $(10.1)$ by an expansion for $c_{\lambda}(n)$ with an error term corresponding to that in (1.12). The details are complicated and we omit them.

We use Cauchy's theorem to prove Theorem 5. If $\Gamma$ is a contour enclosing the points $x=0$ and $x=\lambda$, we have

$$
c_{\lambda}(n)=\frac{1}{2 \pi i} \int_{\Gamma} \frac{f(x+\lambda) d x}{x^{n+1}}=\frac{1}{2 \pi i} \int_{\Gamma} \frac{f(x) d x}{(x-\lambda)^{n+1}} .
$$

11. The numbers $x_{0}$ and $\Lambda\left(X_{0}\right)$. We write $U=U(X)$ putting $Y=X$ in $\S 4$. In Lemma 5 let us take

$$
\vartheta(z)=\lambda(\kappa n)^{-\kappa}(1+z)^{1-\kappa} \exp \left\{\sum a_{j} b_{j} n^{b_{j}-1}(1+z)^{b_{j-1}}\right\}
$$

so that

$$
|\vartheta(z)|<K_{4} n^{-\kappa^{\prime}}<K_{1}=|z|
$$

on the circumference of $A$ when $n>K$. We define $Z$ as in Lemma 5 and write

$$
U_{0}=\kappa n(1+Z), \quad X_{0}=U_{0} \exp \left(-\sum A_{j} b_{j} U_{0}^{b_{j}-1}\right), \quad x_{0}=X_{0}^{\kappa} .
$$

When $U=U_{0}$, we have $X=X_{0}$ and $x=x_{0}$. Also

$$
U_{0}-\kappa n=\kappa n Z=\kappa n \vartheta(Z)=\lambda U_{0}^{1-\kappa} \exp \left(\kappa \sum A_{j} b_{j} U_{0}^{b_{j}-1}\right)=\lambda U_{0} X_{0}^{-\kappa},
$$

and

$$
X_{0}^{\kappa}-\lambda=\frac{\lambda \kappa n}{U_{0}-\kappa n}=\frac{\lambda}{Z}=\frac{\lambda}{\vartheta(Z)}
$$


When the conditions of Theorem 1 are satisfied,

$$
\begin{aligned}
\log \left\{\frac{f(x)}{(x-\lambda)^{n}}\right\} & \sim \phi\{U(X)\}-n \log (x-\lambda) \\
& =\phi(U)-n \log \left(X^{\kappa}-\lambda\right) \\
& =\Lambda(X) \text { (say). }
\end{aligned}
$$

By (4.10),

$$
\frac{d}{d X} \Lambda(X)=\frac{d}{d X} \phi(U)-\frac{\kappa n X^{\kappa-1}}{X^{\kappa}-\lambda}=\frac{U}{X}-\frac{\kappa n X^{\kappa-1}}{X^{\kappa}-\lambda}
$$

and this vanishes for $X=X_{0}$ by (11.3). Hence $x=x_{0}$ is a saddle-point of the integral (10.2).

We now obtain an asymptotic evaluation of $\Lambda\left(X_{0}\right)$ for large $n$. By (11.4),

$$
-n \log \left(X_{0}^{\kappa}-\lambda\right)=n\{\log \vartheta(Z)-\log \lambda\}
$$

and so, by (4.2) and (11.1),

$$
\begin{aligned}
\Lambda\left(X_{0}\right)= & U_{0}+\sum A_{j}\left(1-b_{j}\right) U_{0}^{b_{j}}+n\{\log \vartheta(Z)-\log \lambda\} \\
= & \kappa n\{1-\log (\kappa n)\}+\kappa n Z+n(1-\kappa) \log (1+Z) \\
& +\sum a_{j} n^{b_{j}}\left\{\left(1-b_{j}\right)(1+Z)^{b_{j}}+b_{j}(1+Z)^{b_{j}-1}\right\} .
\end{aligned}
$$

We take this last expression as $p(Z)$ in Lemma 5 and so have

$$
\begin{aligned}
& p(0)=\kappa n-\kappa n \log (\kappa n)+\psi(n), \\
& p^{\prime}(z)=\frac{n(1+\kappa z)}{1+z}+z \sum a_{j} b_{j}\left(1-b_{j}\right) n^{b_{j}}(1+z)^{b_{j}-2} .
\end{aligned}
$$

Differentiating (11.1) logarithmically with respect to $z$, we find that

$$
p^{\prime}(z)=n\left(1-\frac{z \vartheta^{\prime}}{\vartheta}\right)
$$

Now, for $m \geqq 1$,

$$
\begin{aligned}
\frac{d^{m-1}}{d z^{m-1}}\left(z \vartheta^{\prime} \vartheta^{m-1}\right) & =\frac{1}{m} \frac{d^{m-1}}{d z^{m-1}}\left(z \frac{d}{d z} \vartheta^{m}\right) \\
& =\frac{z}{m} \frac{d^{m}}{d z^{m}} \vartheta^{m}+\left(\frac{m-1}{m}\right) \frac{d^{m-1}}{d z^{m-1}} \vartheta^{m}
\end{aligned}
$$

and so, by (11.7),

$$
\left[\frac{d^{m-1}}{d z^{m-1}}\left\{p^{\prime}(z) \vartheta^{m}\right\}\right]_{z=0}=n\left[\frac{d^{m-1}}{d z^{m-1}}\left(\vartheta^{m}-z \vartheta^{\prime} \vartheta^{m-1}\right)\right]_{z=0}=\frac{n}{m}\left[\frac{d^{m-1}}{d z^{m-1}} \vartheta^{m}\right]_{z=0} .
$$


Hence, by Lemma 5,

$$
\Lambda\left(X_{0}\right)=\kappa n-\kappa n \log (\kappa n)+\psi(n)+\sum_{m=1}^{\infty} \mu(m, n),
$$

where

We have

$$
\mu(m, n)=\frac{n}{m(m !)}\left[\frac{d^{m-1}}{d z^{m-1}} \vartheta^{m}\right]_{z=0}
$$

$$
|\mu(m, n)|=\left|\frac{n}{2 \pi i m^{2}} \int \mathcal{A} \frac{\vartheta^{m}}{z^{m}} d z\right| \leqq \frac{K_{4}^{m} n^{1-m k^{\prime}}}{m^{2} K_{1}^{m-1}}
$$

by (11.2) and so

$$
\sum_{m=\left[1 / \kappa^{\prime}\right]+1}^{\infty} \mu(m, n)=O\left(n^{-K}\right)
$$

Again, for bounded $m$ and $z$, since

$$
m \kappa+\left(S_{m}+1\right)(1-b)>1+K,
$$

we have

$$
\begin{aligned}
\vartheta^{m} & =\lambda^{m}(\kappa n)^{-m \kappa}(1+z)^{m(1-\kappa)} \exp \left\{m \sum a_{j} b_{j} n^{b_{j}-1}(1+z)^{b_{j}-1}\right\} \\
& =\lambda^{m}(\kappa n)^{-m \kappa}\left\{(1+z)^{m(1-k)}+\sum_{s=1}^{S_{m}} m^{*} \sum_{(s)} T n^{-\tau}(1+z)^{m-m \kappa-\tau}\right\}+O\left(n^{-1-K}\right)
\end{aligned}
$$

by (2.3) and so

$$
\mu(m, n)=\frac{n}{2 \pi i m^{2}} \int \mathcal{A} \frac{\vartheta^{m}}{z^{m}} d z=n^{1-m \ltimes} \lambda^{m} \psi_{m}(n)+O\left(n^{-K}\right) .
$$

Hence

$$
\begin{aligned}
\Lambda\left(X_{0}\right) & =\kappa n-\kappa n \log (\kappa n)+\psi(n)+\lambda \psi(n, \lambda)+O\left(n^{-K}\right) \\
& =\kappa n-\kappa n \log (\kappa n)+O\left(n^{B}\right) .
\end{aligned}
$$

Similar calculations enable us to show that

$$
U_{0}=\kappa n+O\left(n^{B}\right), \quad X_{0}=\kappa n+O\left(n^{B}\right),
$$

whence

$$
\arg X_{0}=\gamma+O\left(n^{B-1}\right) \text {. }
$$

12. Proof of Theorem 5. By definition the $\mu_{1}, \mu_{2}$ of Theorem 5 satisfy (2.5). We may also suppose that, if $\rho^{\prime}<1 / 2$, then $\mu_{1}<\bar{\mu}_{0}$ and $\mu_{2}<\mu_{0}$. We write 


$$
\mu_{3}=\min \left(\mu_{1}, \omega_{2}, \pi / 2-2 \gamma\right)-\epsilon, \quad \mu_{4}=\min \left(\mu_{2}, \omega_{1}, \pi / 2+2 \gamma\right)-\epsilon,
$$

where $\epsilon$ is a positive number chosen small enough to ensure that

$$
-\mu_{3}<\gamma<\mu_{4}
$$

This is always possible since

$$
-\min \left(\mu_{1}, \omega_{2}\right)<\gamma<\min \left(\mu_{2}, \omega_{1}\right)
$$

by (2.5) and Lemma 3 and

$$
-(\pi / 2-2 \gamma)<\gamma<\pi / 2+2 \gamma,
$$

the latter condition being equivalent to $-\pi / 2<\gamma<\pi / 2$.

We now take $\Gamma$ in (10.2) to be the circle on which $|x|=\left|x_{0}\right|$, write $x=x_{0} e^{i \xi}, X=X_{0} e^{i p \xi}$ on $\Gamma$ and $\eta_{0}=\arg X_{0}$ and choose $\xi$ so that

$$
\frac{\mu_{4}-\eta_{0}}{\rho^{\prime}}-2 \pi<\xi \leqq \frac{\mu_{4}-\eta_{0}}{\rho^{\prime}} \text {. }
$$

We divide $\Gamma$ into two parts $\Gamma_{1}, \Gamma_{2}$ on which

$$
\frac{\mu_{4}-\eta_{0}}{\rho^{\prime}}-2 \pi<\xi<-\left(\frac{\mu_{3}+\eta_{0}}{\rho^{\prime}}\right), \quad-\left(\frac{\mu_{3}+\eta_{0}}{\rho^{\prime}}\right) \leqq \xi \leqq \frac{\mu_{4}-\eta_{0}}{\rho^{\prime}}
$$

respectively, and write

$$
c_{1}=\frac{1}{2 \pi i} \int_{\mathrm{r}_{1}} \frac{f(x) d x}{(x-\lambda)^{n+1}}
$$

and similarly for $c_{2}$ so that $c_{\lambda}(n)=c_{1}+c_{2}$.

LEMMA 15. $\log \left|c_{1}\right|<R\left\{\Lambda\left(X_{0}\right)\right\}-K n$.

Our choice of $\arg x$ and $\arg X$ above differs from that of $\$ 2$ when $x$ lies on part of $\Gamma_{1}$. In Lemma 7, however, there is no restriction on arg $Y$ (except boundedness) and so we may put $Y=X$ in that lemma. Since

$$
-\mu_{1}<-\mu_{3}<\gamma<\mu_{4}<\mu_{2},
$$

we may replace $\mu_{1}, \mu_{2}$ in Lemma 7 by $\mu_{3}, \mu_{4}$. Hence, by (5.6),

$$
f(x)=O\left\{\bar{H}\left(-\mu_{3}, X\right)\right\}+O\left\{\bar{H}\left(\mu_{4}, X e^{2 \pi i \rho}\right)\right\}+O\left(X^{K}\right) .
$$

On $\Gamma,|X|=\left|X_{0}\right| e^{\rho^{\prime} \xi \tan \gamma}$ and $\arg X=\eta_{0}+\rho^{\prime} \xi$. By (11.10), $R\left(X_{0}\right)>K\left|X_{0}\right|$ and so on $\Gamma$

$$
X^{K}=O\left(X_{0}^{K}\right)=O\left(e^{R\left(x_{0}\right)-K\left|x_{0}\right|}\right) .
$$

By Lemma 12, 


$$
\begin{aligned}
\log \bar{H}\left(-\mu_{3}, X\right) & <|X| \cos \mu_{3} \exp \left\{\tan \mu_{3}\left(\mu_{3}+\arg X\right)\right\}+O\left(X^{1-K}\right) \\
& =\left|X_{0}\right| \Omega_{1}(\xi)+O\left(X_{0}^{1-K}\right),
\end{aligned}
$$

where

$$
\Omega_{1}(\xi)=\cos \mu_{3} \exp \left\{\left(\mu_{3}+\eta_{0}+\rho^{\prime} \xi\right) \tan \mu_{3}+\rho^{\prime} \xi \tan \gamma\right\} .
$$

Since $\tan \mu_{3}+\tan \gamma>0$ by (12.2), $\Omega_{1}(\xi)$ increases with $\xi$ and so, on $\Gamma_{1}$,

$$
\begin{aligned}
\Omega_{1}(\xi) & <\Omega_{1}\left\{-\left(\mu_{3}+\eta_{0}\right) / \rho^{\prime}\right\}=\cos \mu_{3} e^{-\left(\mu_{8}+\eta_{0}\right) \tan \gamma} \\
& \left.=e^{-\eta_{0} \tan \gamma}\left\{\cos \gamma e^{\gamma \tan \gamma}-\int_{-\mu_{8}}^{\gamma}(\tan \gamma-\tan \theta) \cos \theta e^{\theta \tan \gamma} d \theta\right)\right\} \\
& <e^{-\eta_{0} \tan \gamma}\left(\cos \gamma e^{\gamma \tan \gamma}-K_{5}\right),
\end{aligned}
$$

where $K_{6}$ depends only on $\gamma$ and $\mu_{3}$. But, by (11.11), $\eta_{0} \sim \gamma$ and so

$$
\begin{aligned}
\Omega_{1}(\xi) & <\cos \eta_{0}-K, \\
\log \bar{H}\left(-\mu_{3}, X\right) & <R\left(X_{0}\right)-K\left|X_{0}\right| .
\end{aligned}
$$

Similar calculations enable us to find the same upper bound for log $\bar{H}\left(\mu_{4}, X e^{2 \pi i \rho}\right)$ on $\Gamma_{1}$. Hence, on $\Gamma_{1}$,

$$
\log |f(x)|<R\left(X_{0}\right)-K\left|X_{0}\right|=R\left\{\phi\left(U_{0}\right)\right\}-K\left|X_{0}\right|
$$

by (4.12). Since $|x|=\left|x_{0}\right| \sim\left|(\kappa n)^{\kappa}\right|$ on $\Gamma_{1}$,

$$
\log \left|\left(\frac{x_{0}-\lambda}{x-\lambda}\right)^{n}\right|=n R\left\{\log \left(\frac{1-\lambda x_{0}^{-1}}{1-\lambda x^{-1}}\right)\right\}=O\left(\frac{n}{\left|x_{0}\right|}\right)=O\left(n^{1-K}\right)
$$

and so

$$
\log \left|\frac{f(x)}{(x-\lambda)^{n}}\right|<R\left\{\Lambda\left(X_{0}\right)\right\}-K\left|X_{0}\right|+O\left(n^{1-K}\right)<R\left\{\Lambda\left(X_{0}\right)\right\}-K n
$$

on $\Gamma_{1}$. Lemma 15 follows at once from the definition of $c_{1}$.

LEMMA 16. $c_{2} \sim n^{\beta} e^{\Lambda\left(X_{0}\right)}$.

Let $x$ lie on $\Gamma_{2}$, so that $-\left(\mu_{3}+\eta_{0}\right) \leqq \rho^{\prime} \xi \leqq \mu_{4}-\eta_{0}$, whence

$$
-\mu_{3} \leqq \arg X \leqq \mu_{4},
$$

since $\arg X=\eta_{0}+\rho^{\prime} \xi$. Hence on $\Gamma_{2}$ our present choice of $\arg X$ is the same as that of $\$ 2$, the conditions of Theorem 1 are satisfied and

$$
\begin{aligned}
f(x) & \sim 2^{1 / 2} \pi^{1 / 2} \kappa^{-1-\beta} X^{1 / 2+\beta} e^{\phi(U)}, \\
\frac{f(x)}{(x-\lambda)^{n}} & \sim 2^{1 / 2} \pi^{1 / 2} \kappa^{-1-\beta} X^{1 / 2+\beta} e^{\Lambda(X)},
\end{aligned}
$$

and 


$$
\log \left\{\frac{f(x)}{(x-\lambda)^{n}}\right\} \sim \Lambda(X)
$$

We put

$$
\Lambda(X)-\Lambda\left(X_{0}\right)=Q(\xi)=Q_{1}(\xi)+i Q_{2}(\xi),
$$

where $Q_{1}(\xi), Q_{2}(\xi)$ are real. By (11.6),

$$
Q^{\prime}(\xi)=i \rho X \frac{d}{d X} \Lambda(X)=i \rho\left(U-\frac{\kappa n x}{x-\lambda}\right)
$$

and

$$
Q^{\prime \prime}(\xi)=-\rho^{2} X \frac{d U}{d X}-\frac{\lambda n x}{(x-\lambda)^{2}}=-\rho^{2} X+O\left(n^{B}\right)
$$

by (4.4) with $d=1$, since $K n<|X|<K n$. Hence

$$
\begin{aligned}
Q_{1}{ }^{\prime \prime}(\xi) & =R\left\{Q^{\prime \prime}(\xi)\right\}=-\left|\rho^{2} X_{0}\right| e^{\rho^{\prime \xi} \tan \gamma} \cos (\arg X-2 \gamma)+O\left(n^{B}\right) \\
& <-K n,
\end{aligned}
$$

since $-\pi / 2+\epsilon \leqq-\mu_{3}-2 \gamma \leqq \arg X-2 \gamma \leqq \mu^{4}-2 \gamma \leqq \pi / 2-\epsilon$ by (12.1) and (12.4).

When $\xi=0$, we have $x=x_{0}, X=X_{0}, U=U_{0}$ and so $Q(0)=0, Q^{\prime}(0)=0$, the last by (11.3). If we apply Taylor's theorem to the two functions $Q_{1}(\xi), Q_{2}(\xi)$ of the real variable $\xi$, we have therefore

$$
Q_{1}(\xi)=\xi^{2} Q_{1}^{\prime \prime}\left(w_{1} \xi\right) / 2, \quad Q_{2}(\xi)=\xi^{2} Q_{2}^{\prime \prime}\left(w_{2} \xi\right) / 2,
$$

where $0<w_{1}, w_{2}<1$. Hence

$$
Q_{1}(\xi)<-K n \xi^{2}
$$

by (12.8).

We choose $\epsilon_{1}$ so that $0<\epsilon_{1}<\min \{1 / 6,(1-B) / 2\}$, write $h=n^{e_{1}-1 / 2}$ and divide $\Gamma_{2}$ into two parts $\Gamma_{3}, \Gamma_{4}$ such that $|\xi|>h$ on $\Gamma_{3}$ and $|\xi| \leqq h$ on $\Gamma_{4}$. We write $c_{2}=c_{3}+c_{4}$ to correspond. On $\Gamma_{3}$, by (12.10),

$$
R\{\Lambda(X)\}=R\left\{\Lambda\left(X_{0}\right)\right\}+Q_{1}(\xi)<R\left\{\Lambda\left(X_{0}\right)\right\}-K n^{2 \epsilon_{1}}
$$

and so, by (12.5),

$$
\left|c_{3}\right|<K\left|n^{1 / 2+\beta} e^{\Lambda\left(X_{0}\right)-K n^{2 \epsilon_{1}}}\right|<K\left|n^{\beta-K^{\Lambda}} e^{\Lambda\left(X_{0}\right)}\right| .
$$

On $\Gamma_{4}$, by (12.7),

$$
\begin{aligned}
Q^{\prime \prime}(\xi) & =-\rho^{2} X+O\left(n^{B}\right)=-\rho^{2} X_{0}+O\left(X_{0} \xi\right)+O\left(n^{B}\right) \\
& =-\rho n+O(n \xi)+O\left(n^{B}\right)
\end{aligned}
$$

and so by (12.9), 


$$
\begin{aligned}
Q(\xi) & =-\rho n \xi^{2} / 2+O\left(n \xi^{3}\right)+O\left(n^{B} \xi^{2}\right) \\
& =-\rho n \xi^{2} / 2+O\left(n^{3 \epsilon 1-1 / 2}\right)+O\left(n^{2 \epsilon 1+B-1}\right) \\
& =-\rho n \xi^{2} / 2+O\left(n^{-K}\right) .
\end{aligned}
$$

Again, on $\Gamma_{4}, x=x_{0} e^{i \xi} \sim x_{0}, X \sim X_{0} \sim \kappa n$ and so, by (12.5),

$$
\begin{aligned}
c_{4} & =\frac{1}{2 \pi i} \int_{\Gamma_{4}} \frac{f(x) d x}{(x-\lambda)^{n+1}}=\frac{n^{1 / 2+\beta} e^{\Lambda\left(X_{0}\right)}}{(2 \pi \kappa)^{1 / 2}} \int_{-h}^{h} e^{Q(\xi)}\left\{1+O\left(n^{-K}\right)\right\} d \xi \\
& =\frac{n^{1 / 2+\beta} e^{\Lambda\left(X_{0}\right)}}{(2 \pi \kappa)^{1 / 2}} \int_{-h}^{h} e^{-\rho n \xi^{2} / 2}\left\{1+O\left(n^{-K}\right)\right\} d \xi \\
& \sim n^{\beta} e^{\Lambda\left(X_{0}\right)}
\end{aligned}
$$

by Lemma 9, with $y=\rho n$. Lemma 16 follows from this and (12.11).

Theorem 5 is an immediate consequence of Lemmas 15 and 16 and (11.8). 13. Proof of Theorem 6.

LEMMA 17. If $\kappa^{\prime} \geqq 1$ and if

$$
c_{0}(t)=O\left\{t^{\sigma} e^{\psi(t)}(e / \kappa t)^{\kappa t}\right\}
$$

for all integral $t \geqq n$, then $c_{\lambda}(n)=O\left\{n^{\sigma} e^{\psi(n)}(e / \kappa n)^{\star n}\right\}$.

In what follows $l$ is a non-negative integer and the $K$ are always independent of $l$. By (1.1) and (1.2)

$$
c_{\lambda}(n)=\sum_{l=0}^{\infty}\left(\begin{array}{c}
n+l \\
l
\end{array}\right) c_{0}(n+l) \lambda^{l}
$$

Let

$$
g(l)=\left(\begin{array}{c}
n+l \\
l
\end{array}\right)\left|(n+l)^{\sigma} e^{\psi(n+l)} \lambda^{l}\left(\frac{e}{\kappa(n+l)}\right)^{\kappa n+\kappa l}\right| .
$$

Now $\psi(n+l+1)-\psi(n+l)=O\left\{(n+l)^{b-1}\right\}, e^{\psi(n+l+1)} \sim e^{\psi(n+l)}$ and so

$$
\left|\frac{g(l+1)}{g(l)}\right| \leqq \frac{K(n+l+1)^{1-\alpha^{\prime}}}{l+1}\left(1+\frac{1}{n+l}\right)^{-x^{\prime}(n+l)} \leqq \frac{K_{7}}{l+1},
$$

since $\kappa^{\prime} \geqq 1$. Hence, by (13.1) and (13.2), we have the result of the lemma

$$
\left|c_{\lambda}(n)\right| \leqq K \sum_{l=0}^{\infty} g(l) \leqq K g(0) \sum_{l=0}^{\infty} \frac{K_{7}^{l}}{l !}<K g(0) .
$$

To prove Theorem 6 we use the $c_{0}^{(1)}(n), c_{0}^{(2)}(n), f^{(1)}(x), f^{(2)}(x)$ of $\S 8$ and write

$$
f^{(p)}(x+\lambda)=\sum_{n=0}^{\infty} c_{\lambda}^{(p)}(n) x^{n} \quad(p=1,2) .
$$


By Theorem 5,

$$
c_{\lambda}^{(1)}(n) \sim e^{\lambda \psi(n, \lambda)} c_{0}^{(1)}(n)
$$

and by the hypothesis of Theorem 6 ,

$$
c_{0}^{(2)}(n)=O\left\{n^{-K_{8}} c_{0}^{(1)}(n)\right\} .
$$

But, since $\kappa^{\prime} \geqq 1, \lambda \psi(n, \lambda)=O(1)$. Hence, applying Lemma 17 to $c_{0}^{(2)}(n)$ with $\sigma=\beta-K_{8}$, we have

$$
c_{\lambda}^{(2)}(n)=O\left\{n^{-K_{8}} \quad c_{0}^{(1)}(n)\right\},
$$

which, combined with (13.4), gives us Theorem 6 .

If $\kappa^{\prime}>1$, the use of Theorem 5 is unnecessary. We have $\psi(n, \lambda)=0$ and

$$
\left|\frac{g(l+1)}{g(l)}\right| \leqq \frac{K(n+l+1)^{-K}}{l+1},
$$

so that (13.2) is an asymptotic series and $c_{\lambda}(n) \sim c_{0}(n)$. Thus, for $\kappa^{\prime}>1$, it is possible to prove Theorem 6 by quite elementary methods. I have found the same to be true when $\kappa=1$ but not, so far as I can discover, when $\kappa^{\prime}=1$ and $\kappa \neq 1$.

14. Proof of Theorem 7. We take $\delta>0$ and define $c_{0}^{(p)}(t)(p=3,4,5)$ by

$$
c_{0}^{(3)}(t)=t^{\delta} e^{\pi t i} c_{0}^{(4)}(t)=t^{\delta} c_{0}^{(5)}(t)=t^{\beta}(e / \kappa t)^{\kappa t},
$$

when $R(t)>0$; also $c_{0}^{(p)}(0)=0$. We define $f^{(p)}(x)$ and $c_{\lambda}^{(p)}(n)$ to correspond. Since $c_{0}^{(4)}(n)=(-1)^{n} c_{0}^{(5)}(n)$, we have

$$
f^{(4)}(x)=f^{(5)}(-x), \quad f^{(4)}(x+\lambda)=f^{(5)}(-x-\lambda)
$$

and so $c_{-\lambda}^{(4)}(n)=(-1)^{n} c_{\lambda}^{(5)}(n)$. If we apply Theorem 5 to $c_{0}^{(3)}(n)$ with $\beta, \lambda$ unchanged and to $c_{0}^{(5)}(n)$ with $\beta-\delta,-\lambda$ replacing $\beta, \lambda$, we obtain

and so

$$
\begin{aligned}
c_{\lambda}^{(3)}(n) & \sim n^{\beta} e^{\lambda \psi(n, \lambda)}(e / \kappa n)^{\kappa n}, \\
c_{\lambda}^{(4)}(n) & =(-1)^{n} c_{-\lambda}^{(5)}(n) \sim n^{\beta-\beta} e^{\pi n i-\lambda \psi(n,-\lambda)}(e / \kappa n)^{\kappa n}
\end{aligned}
$$

$$
c_{\lambda}^{(3)}(n)+c_{\lambda}^{(4)}(n) \sim n^{\beta}(e / \kappa n)^{\kappa n}\left\{e^{\lambda \psi(n, \lambda)}+n^{-\delta} e^{-\lambda \psi(n,-\lambda)-\pi n i}\right\},
$$

where

$$
\psi(n, \lambda)=\kappa^{-\kappa} n^{1-\kappa}\left\{1+O\left(n^{-\kappa}\right)\right\}
$$

But

$$
c_{0}^{(3)}(t)+c_{0}^{(4)}(t) \sim t^{\beta}(e / \kappa t)^{\kappa t}
$$

for large positive $t$. Hence, if Theorem 6 is true for the particular $\kappa$ in question 
and if $c_{0}(t)=c_{0}^{(3)}(t)+c_{0}^{(4)}(t)$, we have $c_{\lambda}^{(3)}(n)+c_{\lambda}^{(4)}(n)=c_{\lambda}(n) \sim n^{\beta} e^{\lambda \psi(n, \lambda)}(e / \kappa n)^{\kappa n}$. But this is only consistent with (14.1) provided that

$$
n^{-\delta} e^{-\lambda \psi(n,-\lambda)}=O\left(n^{-K} e^{\lambda \psi(n, \lambda)}\right)
$$

for every $\lambda$ and suitable $K$. This implies that

$$
R\{-\lambda \psi(n,-\lambda)-\lambda \psi(n, \lambda)\}<K \log n
$$

that is, by (14.2),

$$
R\left\{-2 \lambda \kappa^{-\kappa} n^{1-\kappa}\right\}<K \log n,
$$

which is false for some $\lambda$ unless $\kappa^{\prime} \geqq 1$. Hence the condition $\kappa^{\prime} \geqq 1$ in Theorem 6 is necessary.

\section{BIBLIOGRAPHY}

1. E. W. Barnes, The asymptotic expansion of integral functions defined by Taylor's series, Philos. Trans. Roy. Soc. London, Ser. A vol. 206 (1906) pp. 249-297.

2. W. B. Ford, The asymptotic developments of functions defined by Maclaurin series, University of Michigan Studies, Ann Arbor, 1936.

3. H. K. Hughes, On the asymptotic expansions of entire functions defined by Maclaurin series, Bull. Amer. Math. Soc. vol. 50 (1944) pp. 425-430.

4. - The asymptotic developments of a class of entire functions, Bull. Amer. Math. Soc. vol. 51 (1945) pp. 456-461.

5. C. V. Newsom, On the character of certain entire functions in distant portions of the plane, Amer. J. Math. vol. 60 (1938) pp. 561-572.

6. - The asymptotic behaviour of a class of entire functions, Amer. J. Math. vol. 65 (1943) pp. 450-454.

7. G. N. Watson, $A$ class of integral functions defined by Taylor's series, Trans. Cambridge Philos. Soc. vol. 22 (1913) pp. 15-37.

8. E. T. Whittaker and G. N. Watson, Modern analysis, 3d ed., Cambridge, 1920.

9. E. M. Wright, The asymptotic expansion of the generalised hypergeometric function, J. London Math. Soc. vol. 10 (1935) pp. 286-293.

10. - The asymptotic expansion of the generalised Bessel function, Proc. London Math. Soc. (2) vol. 38 (1935) pp. 257-270.

11. - The asymptotic expansion of integral functions defined by Taylor series, Philos. Trans. Roy. Soc. London Ser. A vol. 238 (1940) pp. 423-451.

12. - The asymptotic expansion of the generalised hypergeometric function, Proc. London Math. Soc. (2) vol. 46 (1940) pp. 389-408.

13. - The generalised Bessel function of order greater than one, Quart. J. Math. Oxford Ser. vol. 11 (1940) pp. 36-48.

14. - The asymptotic expansion of integral functions defined by Taylor series (second paper), Philos. Trans. Roy. Soc. London Ser. A vol. 239 (1941) pp. 217-232.

UNIVERSity OF ABERDEEN, Aberdeen, Scotland. 